\title{
REFLECTIONS ON LOWER BOUNDED LATTICES
}

\author{
K. V. ADARICHEVA AND J. B. NATION \\ In memory of Ivan Rival
}

\begin{abstract}
Lattices in the variety $\mathcal{L} \mathcal{B}(k)$, lower bounded lattices of rank $k$, are characterized. A sufficient condition for a lattice to be lower bounded is given, and used to produce a new example of a non-finitely-generated lower bounded lattice. Lattices that are subdirect products of finite lower bounded lattices are characterized.
\end{abstract}

\section{INTRODUCTION}

The study of bounded lattices and their generalizations began in the early 1970s with the work of McKenzie [9] and Jónsson [6] on projective lattices and lattice varieties; see also Kostinsky [8]. In the ensuing years, finite bounded lattices, and more generally finitely generated lower bounded lattices, came to be well understood. These lattices inherit many structural properties of finitely generated free lattices. Most of the relevant facts concerning finitely generated lower bounded lattices are gathered in the monograph by Freese, Ježek and Nation [4].

While finitely generated lattices form the natural setting for lower bounded homomorphisms, this restriction is artificial for some other purposes. Thus the notion has been extended by defining a lattice $\mathbf{L}$ to be lower bounded if every finitely generated sublattice of $\mathbf{L}$ is lower bounded, i.e., a lower bounded homomorphic image of a free lattice. This class of lattices is denoted by $\mathcal{L B}$.

Finitely generated lower bounded lattices admit a nice characterization: A finitely generated lattice $\mathbf{L}$ is lower bounded if and only if $\mathbf{D}(\mathbf{L})=\mathbf{L}$. The motivation for the current work was to find a similar characterization of lower bounded lattices in general. We have not succeeded, and the problem remains open. There is a natural generalization of the subset $\mathrm{D}(\mathbf{L})$ from Adaricheva and Semenova [3], which is denoted $\mathrm{D}^{*}(\mathbf{L})$; see Definition 3.5. The condition $\mathrm{D}^{*}(\mathbf{L})=\mathbf{L}$ is sufficient, but not necessary, for lower boundedness.

The source of our difficulties is that the class $\mathcal{L B}$ is not closed under direct products (Adaricheva and Gorbunov [2]). One way to avoid this problem is to consider

Date: January 31, 2005.

1991 Mathematics Subject Classification. 06B20, 06B05.

Key words and phrases. lattice, lower bounded lattice, join semidistributive lattice, variety, reflection. 
the varieties $\mathcal{L} \mathcal{B}(k)$, for $k \in \omega$, consisting of all lattices $\mathbf{L}$ such that every finitely generated sublattice $\mathbf{S}$ of $\mathbf{L}$ is finite and all join irreducible elements of $\mathbf{S}$ belong to $\mathrm{D}_{k}(\mathbf{S})$ (from Nation [10]). In Corollary 3.31, we prove that a lattice $\mathbf{L}$ is in $\mathcal{L} \mathcal{B}(k)$ if and only if every completely join irreducible filter of $\mathbf{L}$ is in $\mathrm{D}_{k}^{*}(\mathcal{F}(\mathbf{L}))$, where $\mathcal{F}(\mathbf{L})$ is the filter lattice of $\mathbf{L}$. Even better, in Theorem 3.20 we describe the largest homomorphic image in $\mathcal{L} \mathcal{B}(k)$ of an arbitrary lattice $\mathbf{L}$.

Returning to the more general class $\mathcal{L} \mathcal{B}$, in Theorem 4.4 we give a new sufficient condition for a lattice to be lower bounded. This allows us to build a new example of lower bounded lattice that is not locally finite and does not satisfy the condition $\mathrm{D}^{*}(\mathbf{L})=\mathbf{L}$.

As a prelude, we make a connection between the varieties of lower bounded lattices $\mathcal{L} \mathcal{B}(k)$ and varieties of join semidistributive lattices $\mathcal{S D}_{\vee}(k)$. For a postlude, we characterize lattices that are subdirect products of finite lower bounded lattices.

Let us establish some notation. Given a class $\mathcal{K}$ of algebras and an algebra $\mathbf{A}$, let

$$
\kappa_{\mathcal{K}}^{\mathbf{A}}=\bigcap\{\theta \in \operatorname{Con} \mathbf{A}: \mathbf{A} / \theta \in \mathcal{K}\} .
$$

As long as $\mathcal{K}$ is a quasivariety, then $\mathbf{A} / \kappa_{\mathcal{K}}^{\mathbf{A}} \in \mathcal{K}$, and $\kappa_{\mathcal{K}}^{\mathbf{A}}$ is the least congruence $\theta$ such that $\mathbf{A} / \theta \in \mathcal{K}$. The quotient $\mathbf{A} / \theta$ is called the reflection of $\mathbf{A}$ in $\mathcal{K}$.

For a lattice $\mathbf{L}$, let $\mathrm{J}(\mathbf{L})$ denote the non-zero join irreducible elements of $\mathbf{L}$. For an element $a$ in a lattice $\mathbf{L}$, we use $\uparrow a$ to denote the principal filter generated by $a$. For a subset $U \subseteq \mathbf{L}$, let

$$
\begin{aligned}
U^{\vee} & =\{\bigvee A: A \subseteq U, A \text { finite }\} \\
U^{\vee} & =\{\bigvee A: A \subseteq U\} \\
U^{\wedge} & =\{\bigwedge A: A \subseteq U, A \text { finite }\} \\
U^{\wedge} & =\{\bigwedge A: A \subseteq U\} .
\end{aligned}
$$

The sublattice generated by $U$ is denoted $\operatorname{Sg}(U)$. For subsets $A, B \subseteq \mathbf{L}$ we write $A \ll B$ if for every $a \in A$ there exists $b \in B$ such that $a \leq b$. The subsets $\mathrm{D}_{k}(\mathbf{L})$ are defined recursively. Let $\mathrm{D}_{0}(\mathbf{L})$ be the set of all join prime elements of $\mathbf{L}$, i.e., those elements $a \in \mathbf{L}$ such that $a \leq b \vee c$ implies $a \leq b$ or $a \leq c$. For $k>0$, let $a \in \mathrm{D}_{k}(\mathbf{L})$ if whenever $a \leq \bigvee B$ for a finite subset $B$ of $\mathbf{L}$, then either $a \leq b$ for some $b \in B$, or there exists a finite subset $C \subseteq \mathrm{D}_{k-1}(\mathbf{L})$ such that $C \ll B$ and $a \leq \bigvee C$. Finally, let $\mathrm{D}(\mathbf{L})=\bigcup_{k \geq 0} \mathrm{D}_{k}(\mathbf{L})$.

A homomorphism $h: \mathbf{K} \rightarrow \mathbf{L}$ is said to be lower bounded if for every $a \in \mathbf{L}$, whenever $\{x \in \mathbf{K}: h(x) \geq a\}$ is nonempty, then it has a least element, denoted $\beta_{h}(a)$. Thus $\beta_{h}: \mathbf{L} \rightarrow \mathbf{K}$ is a partial map, called the lower bound map, with the property that for all $x \in \mathbf{L}$ and $a \in \mathbf{K}, h(x) \geq a$ if and only if $h(y) \geq a$ for some $y \in \mathbf{L}$ and $x \geq \beta_{h}(a)$. A finitely generated lattice is lower bounded if it is a lower 
bounded homomorphic image of a free lattice. Recall that a finitely generated lattice $\mathbf{L}$ is lower bounded if and only if $\bigcup_{k \geq 0} \mathrm{D}_{k}(\mathbf{L})=\mathbf{L}$. Recall that $\left(\mathrm{D}_{k}(\mathbf{L})\right)^{\vee} \subseteq \mathrm{D}_{k+1}(\mathbf{L})$, and that each $\mathrm{D}_{k}(\mathbf{L})$ is finite whenever $\mathbf{L}$ is finitely generated. Thus the above definition is equivalent to: $\mathbf{L} \in \mathcal{L} \mathcal{B}(k)$ if every finitely generated sublattice $\mathbf{S}$ of $\mathbf{L}$ satisfies $\mathbf{S} \subseteq \mathrm{D}_{k+1}(\mathbf{S})$ and $\mathrm{J}(\mathbf{S}) \subseteq \mathrm{D}_{k}(\mathbf{S})$.

\section{Classes of LOWER BOUNDED AND JOIN SEMIDistributive LATTICES}

Let $k \in \omega$. Then $\mathcal{L} \mathcal{B}(k)$ is a locally finite variety, and for any finite set $X$, the relatively free lattice $\mathbf{F}_{\mathcal{L B}(k)}(X)$ is isomorphic to $X^{(\wedge \vee)^{k+1}}$ in $\mathbf{F L}(X)$, the free lattice generated by $X$. This is a finite join semilattice with zero, and a lower bounded lattice with the inherited order.

It is well known that lower bounded lattices are join semidistributive, i.e., satisfy the following law:

$$
x \vee y=x \vee z \text { implies that } x \vee y=x \vee(y \wedge z) \text { for all } x, y, z .
$$

Let $S \mathcal{D}_{\vee}(k)$ denote the variety of lattices defined by the law $y_{k+1} \leq x \vee(y \wedge z)$, where

$$
\begin{aligned}
y_{0} & =y \quad z_{0}=z \\
y_{n+1} & =y \wedge\left(x \vee z_{n}\right) \\
z_{n+1} & =z \wedge\left(x \vee y_{n}\right) .
\end{aligned}
$$

It was shown in Jónsson and Rival [7] (see also [10]) that every lattice in a variety $\mathcal{V}$ is join semidistributive if and only if $\mathcal{V} \subseteq S \mathcal{D}_{\vee}(k)$ for some $k \in \omega$.

There is a basic connection between these varieties.

Lemma 2.1. For every $k \in \omega$ we have $\mathcal{L} \mathcal{B}(k) \subseteq \mathcal{S D}_{\vee}(k)$.

Proof. Let $X=\{x, y, z\}$, and let $\sigma_{k}: \mathbf{F L}(X) \rightarrow X^{(\wedge \vee)^{k+1}}$ be the standard homomorphism, given by

$$
\sigma_{k}(w)=\bigvee\left\{u \in X^{(\wedge \vee)^{k+1}}: u \leq w\right\}
$$

We want to show that $\sigma_{0}\left(y_{1}\right)=(x \wedge y) \vee(y \wedge z)$ and, for $k>0$, that $\sigma_{k}\left(y_{k+1}\right)=$ $y \wedge(x \vee(y \wedge z))$, so that $y_{k+1} \leq x \vee(y \wedge z)$ holds in $\mathcal{L} \mathcal{B}(k)$. This follows from a (straightforward) inductive proof of the following two statements (in this order).

I. If $k \geq 1$ and $w \in X^{(\wedge \vee)^{k}}$ and $w \leq x \vee z_{k}$, then $w \leq x \vee(y \wedge z)$.

II. If $k \geq 0$ and $w \in X^{(\wedge \vee)^{k+1}}$ and $w \leq y_{k+1}$, then $w \leq x \vee(y \wedge z)$.

M. Semenova points out that one can also derive the inclusion $y_{k+1} \leq x \vee(y \wedge z)$ from the equations given for $\mathcal{L} \mathcal{B}(k)$ in either [11] or [13].

Of course, $\mathcal{L} \mathcal{B}(k) \subseteq \mathcal{L} \mathcal{B}$. If $\mathbf{L}$ is a finite join semidistributive lattice, then the relatively free lattice $\mathbf{F}_{\mathcal{V}(\mathbf{L})}(3)$ is finite and join semidistributive, whence an easy argument shows that $\mathcal{V}(\mathbf{L}) \subseteq \mathcal{S} \mathcal{D}_{\vee}(k)$ for some $k$. There are finite join semidistributive lattices 
that are not lower bounded, e.g., the lattice of convex subsets of a four-element chain. Thus $\mathcal{S} \mathcal{D}_{\vee}(k) \nsubseteq \mathcal{L} \mathcal{B}$.

Let $\mathbf{N}_{0}$ be a two-element chain, and recursively define lattices $\mathbf{N}_{k+1}=\mathbf{N}_{k} \cup$ $\left\{0_{k+1}, c_{k+1}, 1_{k+1}\right\}$ where $0_{k+1}$ and $1_{k+1}$ are the least and greatest element of $\mathbf{N}_{k+1}$, respectively, and $c_{k+1}$ is a complement of every element of $\mathbf{N}_{k}$. Thus $\mathbf{N}_{1}$ is a pentagon, $\mathbf{N}_{2}$ is a pentagon within a pentagon, etc. Then $\mathbf{N}_{k} \in \mathcal{L} \mathcal{B}(k) \backslash \mathcal{L} \mathcal{B}(k-1)$, but the 3 -generated sublattices of $\mathbf{N}_{k}$ are all in $\mathcal{V}\left(\mathbf{N}_{1}\right)$, the variety of the pentagon, so that $\mathbf{N}_{k} \in \mathcal{S} \mathcal{D}_{\vee}(1)$. In particular, it is not true that $\mathcal{S} \mathcal{D}_{\vee}(k) \cap \mathcal{L} \mathcal{B} \subseteq \mathcal{L} \mathcal{B}(k)$, as one might hope.

The fact that $\mathcal{L B}$ is not a quasivariety can make it difficult to deal with nonfinitely-generated lower bounded lattices. In the next section, we consider reflections in the varieties $\mathcal{L} \mathcal{B}(k)$, before returning to the more general class $\mathcal{L} \mathcal{B}$ in section 4 .

\section{Representation theOREM FOR REFLECTIONS IN $\mathcal{L} \mathcal{B}(k)$}

3.1. The finitely generated case. Let $\lambda_{k}=\kappa_{\mathcal{L} \mathcal{B}(k)}^{\mathbf{L}}$ denote the kernel of the reflection on a lattice $\mathbf{L}$.

Theorem 3.1. If $\mathbf{L}$ is finitely generated, then $\lambda_{k}$ is the kernel of the natural map $\varphi_{k}: \mathbf{L} \rightarrow\left(\mathrm{D}_{k}(\mathbf{L})\right)^{\vee}$ given by $\varphi_{k}(x)=\bigvee\left\{p \in \mathrm{D}_{k}(\mathbf{L}): p \leq x\right\}$.

Proof. There are really several claims here.

(1) $\left(\mathrm{D}_{k}(\mathbf{L})\right)^{\vee} \in \mathcal{L} \mathcal{B}(k)$.

(2) The map $\varphi_{k}: \mathbf{L} \rightarrow\left(D_{k}(\mathbf{L})\right)^{\vee}$ is a homomorphism (whence $\lambda_{k} \leq \operatorname{ker} \varphi_{k}$ ).

(3) If $h: \mathbf{L} \rightarrow \mathbf{K} \in \mathcal{L} \mathcal{B}(k)$ and $h(x) \not \leq h(y)$, then there exists $p \in \mathrm{D}_{k}(\mathbf{L})$ with $p \leq x$ and $p \not \leq y$ (whence $\operatorname{ker} \varphi_{k} \leq \lambda_{k}$ ).

Let $\mathbf{S}=\left(\mathrm{D}_{k}(\mathbf{L})\right)^{\vee}$. A straightforward induction shows that $\mathrm{D}_{j}(\mathbf{L}) \subseteq \mathrm{D}_{j}(\mathbf{S})$ for $j \leq k$. Moreover, $\mathrm{J}(\mathbf{S}) \subseteq \mathrm{D}_{k}(\mathbf{L}) \subseteq \mathrm{D}_{k}(\mathbf{S})$, proving (1). Claim (2) follows because $\mathrm{D}_{k}(\mathbf{L})$ has the weak refinement property; see Lemma 3.23 below. Part (3) follows because $h$ is a lower bounded homomorphism, and the lower bound map $\beta_{h}$ has the property that $a \in \mathrm{D}_{j}(\mathbf{K})$ implies $\beta_{h}(a) \in \mathrm{D}_{j}(\mathbf{L})$. (Theorem 3.1 has been known for many years, though we cannot find it explicitly in the literature.)

However, we note that the finite generation of $\mathbf{L}$ is essential in Theorem 3.1. For example, $\mathrm{D}_{0}(\mathbf{L})$ can be empty in a distributive lattice, as it is in the lattice of cofinite subsets of an infinite set.

Corollary 3.2. If $\mathbf{L}$ is finitely generated, then $\mathbf{L} \in \mathcal{L B}$ if and only if $\bigcap \lambda_{k}=0$.

Proof. For $\mathbf{L}$ finitely generated, $\mathbf{L} \in \mathcal{L B}$ if and only if $\mathbf{L}=\bigcup_{k \geq 0} \mathrm{D}_{k}(\mathbf{L})$.

It would be nice if the corollary were true in general, i.e., for non-finitely-generated lattices. The following two examples demonstrate that this is not the case.

Example 3.3. Shows that "if" part fails. 
Let $\mathbf{L}=\Pi_{k \in \omega} \operatorname{Sub}_{\wedge}(B(k))$, where $B(k)$ is the Boolean lattice with $k$ atoms. We have $\operatorname{Sub}_{\wedge}(B(k+1)) \in \mathcal{L} \mathcal{B}(k) \backslash \mathcal{L} \mathcal{B}(k-1)$. If $\pi_{k}: \mathbf{L} \rightarrow \operatorname{Sub}_{\wedge}(B(k+1))$ is the projection map, then $\lambda_{k}(\mathbf{L}) \subseteq \operatorname{ker} \pi_{k}$. Hence $\bigcap \lambda_{k}=0$.

On the other hand, $\mathbf{L}$ has the ideal lattice of a free lattice as a sublattice, thus it is not lower bounded (see Adaricheva and Gorbunov [2]).

Example 3.4. Shows that "only if" part fails, too.

It is proved in [2] that the lattice $\operatorname{Sub}_{\mathrm{f}}(\mathbf{P})$ of finite subsemilattices of a semilattice $\mathbf{P}$ is lower bounded.

Consider the $\wedge$-semilattice $\mathbf{P}=\omega \times \omega$ and let $\mathbf{L}=\operatorname{Sub}_{\mathrm{f}}(\mathbf{P})$. We want to show that, for any $\theta \in \operatorname{Con}(\mathbf{L})$, if $\mathbf{L} / \theta \in \mathcal{L} \mathcal{B}(k)$ then $\theta=1$. In particular, $\lambda_{k}=1$ for every $k \in \omega$.

Indeed, if $\theta \neq 1$ then $\left\{p_{0}\right\} \not_{\theta} 0$ for some atom $\left\{p_{0}\right\} \in \mathbf{L}$. In the subalgebra lattice of any meet semilattice, if the elements $a$ and $b$ are incomparable, then $\{a\} \equiv_{\theta} 0$ implies $\{a \wedge b\} \equiv_{\theta} 0$. If $q>p$ in $\omega \times \omega$, then $p$ can be obtained from $q$ by a sequence of one or two proper meets. Hence, for $q>p$ in $\mathbf{P},\{q\} \equiv_{\theta} 0$ implies $\{p\} \equiv_{\theta} 0$. It follows that $\{q\} \not \equiv_{\theta} 0$ for every $q>p_{0}$.

Let $\mathbf{P}_{0}=\uparrow p_{0}$ be the principal filter generated by $p_{0}$ in $\mathbf{P}$. For any $\mathbf{S}_{1}, \mathbf{S}_{2} \in \operatorname{Sub}_{\mathrm{f}}\left(\mathbf{P}_{0}\right)$,

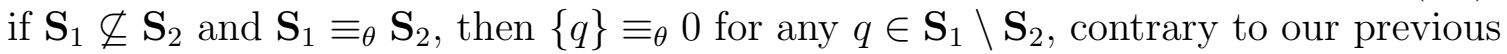
argument. Thus $\mathbf{S}_{1} \equiv{ }_{\theta} \mathbf{S}_{2}$ only if $\mathbf{S}_{1}=\mathbf{S}_{2}$, and so the map $\mathbf{S} \mapsto \mathbf{S} / \theta$ defines an embedding of $\operatorname{Sub}_{\mathrm{f}}\left(\mathbf{P}_{0}\right)$ into $\mathbf{L} / \theta$. It remains to notice that $\operatorname{Sub}_{\mathrm{f}}\left(\mathbf{P}_{0}\right)$ is isomorphic to $\operatorname{Sub}_{\mathrm{f}}(\mathbf{P})$, and that $\operatorname{Sub}_{\mathrm{f}}(\mathbf{P}) \notin \mathcal{L} \mathcal{B}(k)$ for every $k \in \omega$.

3.2. A generalization of $\mathrm{D}_{k}(\mathbf{L})$. In order to formulate the proper generalization of Theorem 3.1, we work in the filter lattice $\mathcal{F}(\mathbf{L})$, which is ordered by reverse set inclusion. Its join operation is set intersection.

Let $\Delta_{0}(\mathbf{L})$ denote the set of prime filters of $\mathbf{L}$, and more generally $\Delta_{k}(\mathbf{L})=$ $\mathrm{D}_{k}(\mathcal{F}(\mathbf{L}))$. For $k=0$ and for the case when $\mathbf{L}$ is finitely generated, $\Delta_{k}(\mathbf{L})$ is the appropriate set of filters to consider. In general, however, we must work with a slightly larger set of filters, which in turn is derived from a slight extension of the sets $\mathrm{D}_{k}(\mathbf{L})$. The following definition was first used in Adaricheva and Semenova [3].

Definition 3.5. For a lattice $\mathbf{L}$, let $\mathrm{D}_{0}^{*}(\mathbf{L})$ denote the set of (finitely) join prime elements of $\mathbf{L}$. Given $\mathrm{D}_{k}^{*}(\mathbf{L})$, define $\mathrm{D}_{k+1}^{*}(\mathbf{L})$ to be the set of all $p \in \mathbf{L}$ such that, for any finite $S \subseteq \mathbf{L}$, if $p \leq \bigvee S$ nontrivially then that there exists a (possibly infinite) set $T \subseteq \mathrm{D}_{k}^{*}(\mathbf{L})$ with $p \leq \bigvee T$ and $T \ll S$.

Let $\mathrm{D}^{*}(\mathbf{L})=\bigcup_{k \geq 0} \mathrm{D}_{k}^{*}(\mathbf{L})$. We say that a lattice $\mathbf{L}$ satisfies the weak Jónsson property if $\mathrm{D}^{*}(\mathbf{L})=\mathbf{L}$. These notions are particularly appropriate for complete lattices, but the definition does not require completeness.

Clearly $\mathrm{D}_{0}^{*}(\mathbf{L})=\mathrm{D}_{0}(\mathbf{L})$, and $\mathrm{D}_{k}^{*}(\mathbf{L}) \supseteq \mathrm{D}_{k}(\mathbf{L})$ for $k>0$. A straightforward argument shows that if $\mathbf{L}$ is complete, then $\left(\mathrm{D}_{k}^{*}(\mathbf{L})\right)^{\vee} \subseteq \mathrm{D}_{k+1}^{*}(\mathbf{L})$, that is, $S \subseteq \mathrm{D}_{k}^{*}(\mathbf{L})$ implies $\bigvee S \in \mathrm{D}_{k+1}^{*}(\mathbf{L})$. 
The usefulness of these ideas is seen in the following result from [3].

Theorem 3.6. Any lattice with the weak Jónsson property is lower bounded.

The proof is just an enhancement of Jónsson's original argument for the condition $\mathrm{D}(\mathbf{L})=\mathbf{L}$; see [6] or [4], Theorem 2.6. Rather than reproduce the proof here, we note that the theorem is a consequence of our next result, which is a refinement of [4], Corollary 2.15, from [10].

Lemma 3.7. Let $\mathbf{K}$ be a finitely generated sublattice of a lattice $\mathbf{L}$. Define a partial map $\sigma: \mathbf{L} \rightarrow \mathbf{K}$ by letting $\sigma(x)$ be the least element of $\uparrow x \cap \mathbf{K}$, whenever such an element exists. Let $k \geq 0$. If $x \in \mathrm{D}_{k}^{*}(\mathbf{L})$ and $\uparrow x \cap \mathbf{K}$ is nonempty, then $\sigma(x) \in \mathrm{D}_{k}(\mathbf{K})$.

Proof. Where defined, $\sigma$ satisfies the following.

(1) $x \leq \sigma(x)$.

(2) $x \in \mathbf{K}$ if and only if $\sigma(x)=x$.

(3) $x \leq y$ implies $\sigma(x) \leq \sigma(y)$.

(4) $\sigma(x \vee y)=\sigma(x) \vee \sigma(y)$.

(5) More generally, if $\bigvee X$ is defined, and $\sigma(x)$ is defined for every $x \in X$, and $\sigma(X)=\{\sigma(x): x \in X\}$ is finite, then $\sigma(\bigvee X)=\bigvee \sigma(X)$

(6) If $y \in \mathbf{K}$ and $\sigma(x)$ is defined, then $x \leq y$ iff $\sigma(x) \leq y$.

Let $\mathbf{K}$ be generated by the finite set $T$. Note that $\uparrow x \cap \mathbf{K} \neq \emptyset$ if and only if $x \leq \bigvee T$. We prove the following two statements by induction on $k$.

(i) If $a \in \mathrm{D}_{k}^{*}(\mathbf{L})$ and $\uparrow a \cap \mathbf{K} \neq \emptyset$, then $\sigma(a)=\bigwedge\left(\uparrow a \cap T^{(\wedge \vee)^{k}}\right)$.

(ii) If $a \in \mathrm{D}_{k}^{*}(\mathbf{L})$ and $\uparrow a \cap \mathbf{K} \neq \emptyset$, then $\sigma(a) \in \mathrm{D}_{k}(\mathbf{K})$.

Part (i) is to make sure that $\sigma(a)$ is defined, whence part (ii) makes the desired claim about that element.

First let $k=0$, so that $a \in \mathrm{D}_{0}^{*}(\mathbf{L})$. If $a \leq \bigvee T$, then because $a$ is join prime, $\uparrow a \cap T \neq \emptyset$. Let $\hat{a}=\bigwedge(\uparrow a \cap T)$, and let $\mathbf{S}=\{w \in \mathbf{K}: w \geq a$ implies $w \geq \hat{a}\}$. Then $\mathbf{S} \supseteq T$ and it is closed under meet and join, so $\mathbf{S}=\mathbf{K}$. That proves (i), i.e., $\sigma(a)=\hat{a}$. If $B \subseteq \mathbf{K}$ and $\sigma(a) \leq \bigvee B$, then $a \leq \bigvee B$ whence $a \leq b_{0}$ for some $b_{0} \in B$. So $\sigma(a) \leq b_{0}$. Thus $\sigma(a) \in \mathrm{D}_{0}(\mathbf{K})$.

Now let $k>0$ and assume that we have proved (i) and (ii) for $k-1$. Let $a \in \mathrm{D}_{k}^{*}(\mathbf{L})$ with $a \leq \bigvee T$, and let $\hat{a}=\bigwedge\left(\uparrow a \cap T^{(\wedge \vee)^{k}}\right)$. Defining $\mathbf{S}$ as above, we have $\mathbf{S} \supseteq T$ and meet closure immediately. Suppose $W$ is a finite subset of $\mathbf{S}$ and $\bigvee W \geq a$. If $a \leq w$ for some $w \in W$, then $\hat{a} \leq w \leq \bigvee W$, as desired. Otherwise, there exists a set $C \subseteq \mathrm{D}_{k-1}^{*}(\mathbf{L})$ such that $C \ll W$ and $a \leq \bigvee C$. For each $c \in C$, if $c \leq w \in W$, then $c \leq \sigma(c) \leq w$ and $\sigma(c) \in T^{(\wedge \vee)^{k-1} \wedge}$. Moreover, there are only finitely many candidates for $\sigma(c)$, even though $C$ may be infinite. Hence $a \leq \bigvee \sigma(C) \in T^{(\wedge \vee)^{k}}$, so $\hat{a} \leq \bigvee \sigma(C) \leq \bigvee W$. This gives join closure, whence $\mathbf{S}=\mathbf{K}$ follows. Finally, suppose $B$ is a finite subset of $\mathbf{K}$ and $\sigma(a) \leq \bigvee B$ nontrivially. Then also $a \leq \bigvee B$ nontrivially, and hence there exists $C \subseteq \mathrm{D}_{k-1}^{*}(\mathbf{L})$ such that $C \ll B$ and $a \leq \bigvee C$. 
Then $\sigma(C) \ll B$ and $\sigma(a) \leq \sigma(\bigvee C)=\bigvee \sigma(C)$, and by induction $\sigma(C) \subseteq \mathrm{D}_{k-1}(\mathbf{K})$. Thus $\sigma(a) \in \mathrm{D}_{k}(\mathbf{K})$, as desired.

Corollary 3.8. If $\mathbf{L}$ is a finitely generated lattice, then $\mathrm{D}_{k}^{*}(\mathbf{L})=\mathrm{D}_{k}(\mathbf{L})$ for every $k \geq 0$.

Let $\mathbf{K}$ be the lattice of all cofinite subsets of an infinite set $X$, and let $\mathbf{F}$ be the filter lattice $\mathcal{F}(\mathbf{K})$. Then any principal filter of $\mathbf{K}$ is in $\mathrm{D}_{1}^{*}(\mathbf{F})$ but not $\mathrm{D}_{1}(\mathbf{F})$, so the containment may be proper for non-finitely-generated lattices.

Lemma 3.7 also gives us an analogue of Theorem 3.6 for $\mathcal{L} \mathcal{B}(k)$.

Corollary 3.9. For any lattice $\mathbf{L}$, if $\left(\mathrm{D}_{k}^{*}(\mathbf{L})\right)^{\vee}=\mathbf{L}$, then $\mathbf{L} \in \mathcal{L} \mathcal{B}(k)$.

Proof. Consider an element $b \in \mathbf{K}$, where $\mathbf{K}$ is a finitely generated sublattice of $\mathbf{L}$. Assume $b=\bigvee_{i \in I} a_{i}$ with each $a_{i} \in \mathrm{D}_{k}^{*}(\mathbf{L})$. By Lemma 3.7, each $\sigma\left(a_{i}\right)$ is in $\mathrm{D}_{k}(\mathbf{K})$, whence there are only finitely many of them. Then $b=\sigma(b)=\bigvee_{i \in I} \sigma\left(a_{i}\right)$. Thus $\mathbf{K}$ is finite and $\mathrm{J}(\mathbf{K}) \subseteq \mathrm{D}_{k}(\mathbf{K})$. We conclude that $\mathbf{L} \in \mathcal{L} \mathcal{B}(k)$.

3.3. Another characterization of $\mathrm{D}_{k}^{*}(\mathcal{F}(\mathbf{L}))$. Let $\Delta_{k}^{*}(\mathbf{L})$ denote $\mathrm{D}_{k}^{*}(\mathcal{F}(\mathbf{L}))$. In order to determine the reflection of a lattice $\mathbf{L}$ into $\mathcal{L} \mathcal{B}(k)$, we need an intrinsic description of these filters. To keep matters straight, we shall define sets of filters $\Gamma_{k}(\mathbf{L})$, with the goal of eventually showing that $\Gamma_{k}(\mathbf{L})=\Delta_{k}^{*}(\mathbf{L})$.

Definition 3.10. Let $\Gamma_{k}(\mathbf{L})$ be the collection of all filters $G \in \mathcal{F}(\mathbf{L})$ with the property that for every $x \in G$ and every finite subset $U \subseteq L$ with $x \in \operatorname{Sg}(U)$ there exists an element $y \in \mathrm{D}_{k}(\operatorname{Sg}(U))$ with $y \leq x$ and $y \in G$.

Lemma 3.11. For a filter $F$ in a lattice $\mathbf{L}$, the following are equivalent.

(1) $F \in \Gamma(\mathbf{L})$.

(2) For every finite subset $U \subseteq \mathbf{L}$ such that $\bigvee U \in F$, there is an element $y \in$ $F \cap \mathrm{D}_{k}(\mathbf{L})$ such that $\left.F\right|_{\operatorname{Sg}(U)}=\left.\uparrow y\right|_{\operatorname{Sg}(U)}$.

Proof. If $F \in \Gamma_{k}(\mathbf{L})$ and $U$ is a finite subset of $\mathbf{L}$, then $\mathrm{D}_{k}(\operatorname{Sg}(U))$ is finite, so we can take $y=\bigwedge\left(F \cap \mathrm{D}_{k}(\operatorname{Sg}(U))\right)$. Thus (1) implies (2), and the reverse implication is obvious.

Recall that $\Delta_{0}^{*}(\mathbf{L})$ is the set of filters that are join prime in $\mathcal{F}(\mathbf{L})$.

Lemma 3.12. Let $\mathbf{L}$ be a lattice and $P \in \mathcal{F}(\mathbf{L})$. The following are equivalent.

(1) $P \in \Delta_{0}^{*}(\mathbf{L})$.

(2) $P \in \Gamma_{0}(\mathbf{L})$.

(3) $P$ is a prime filter, i.e., $s \vee t \in P$ implies $s \in P$ or $t \in P$.

Proof. The equivalence of (1) and (3) is straightforward.

To show that (3) implies (2), we assume that $P$ is a prime filter and $x \in \mathbf{L}$. Suppose $U$ is finite and $x \in \operatorname{Sg}(U)$. Then the restriction of $P$ to $\operatorname{Sg}(U)$ is a prime filter in a 
finitely generated lattice, and hence principal, say $\left.P\right|_{\operatorname{Sg}(U)}=\left.\uparrow y\right|_{\operatorname{Sg}(U)}$. Moreover, this $y$ is join prime in $\operatorname{Sg}(U)$. Thus $P \in \Gamma_{0}(\mathbf{L})$.

Conversely, let $P \in \Gamma_{0}(\mathbf{L})$ and suppose $s_{1} \vee s_{2} \in P$. Let $x=s_{1} \vee s_{2}$ and let $U=$ $\left\{s_{1}, s_{2}\right\}$. By the definition of $\Gamma_{0}(\mathbf{L})$, there exists $y \leq s_{1} \vee s_{2}$ such that $y \in \mathrm{D}_{0}(\operatorname{Sg}(U))$ and $y \in P$. Clearly $y=s_{1}$ or $y=s_{2}$, whence $s_{1} \in P$ or $s_{2} \in P$, as desired.

Lemma 3.13. For any lattice $\mathbf{L}$ and any $k \geq 0$ we have $\Delta_{k}^{*}(\mathbf{L}) \subseteq \Gamma_{k}(\mathbf{L})$.

Proof. We will prove by induction on $k$ that $\Delta_{k}^{*}(\mathbf{L}) \subseteq \Gamma_{k}(\mathbf{L})$. The case $k=0$ follows from the previous lemma.

So assume that $k>0$ and $F \in \Delta_{k}^{*}(\mathbf{L})$. Let $U$ be a finite subset of $\mathbf{L}$, and let $x \in F \cap \operatorname{Sg}(U)$. Let $p=\bigwedge\left(F \cap U^{\wedge(\vee \wedge)^{k}}\right)$, and let $y=p \wedge x$. We want to show that $y \in \mathrm{D}_{k}(\operatorname{Sg}(U))$.

Suppose $y \leq \bigvee_{i=1}^{m} w_{i}$ nontrivially with each $w_{i} \in \operatorname{Sg}(U)$. Then $F \leq \bigvee_{i=1}^{m} \uparrow w_{i}$ nontrivially. Since $F \in \Delta_{k}^{*}(\mathbf{L})$, this refines to a cover $F \leq \bigvee_{j \in J} H_{j}$ in $\mathcal{F}(\mathbf{L})$ with each $H_{j} \in \Delta_{k-1}^{*}(\mathbf{L})$. In particular, for each $j$ there exists an $i_{j}$ with $w_{i_{j}} \in H_{j}$. By induction, each $H_{j}$ is in $\Gamma_{k-1}(\mathbf{L})$, so by Lemma 3.11(2) the restriction $\left.H_{j}\right|_{\operatorname{Sg}(U)}$ is principal, say $\left.H_{j}\right|_{\operatorname{Sg}(U)}=\left.\uparrow h_{j}\right|_{\operatorname{Sg}(U)}$ with $h_{j} \in \mathrm{D}_{k-1}(\operatorname{Sg}(U))$ and $h_{j} \leq w_{i_{j}}$. Then also $h_{j} \in U^{\wedge(\vee \wedge)^{k-1}}$, so that there are only finitely many candidates for the elements $h_{j}$, $j \in J$. Thus $y=p \wedge x \leq p \leq \bigvee_{j} h_{j}$. We have produced a (finite) refinement in $\mathrm{D}_{k-1}(\operatorname{Sg}(U))$ of the original cover of $y$, so $y \in \mathrm{D}_{k}(\operatorname{Sg}(U))$. Hence $F \in \Gamma_{k}(\mathbf{L})$. (Moreover, from $y \in \mathrm{D}_{k}(\operatorname{Sg}(U))$ it follows that $y \in U^{\wedge(\vee \wedge)^{k}}$, whence $y=p$. Thus $p$ is the least element of $\left.F\right|_{\operatorname{Sg}(U)}$.)

Let us pause at this point to complete the finitely generated case.

Lemma 3.14. If $\mathbf{L}$ is finitely generated, then the following are equivalent for $F \in$ $\mathcal{F}(\mathbf{L})$.

(1) $F \in \Delta_{k}(\mathbf{L})$.

(2) $F \in \Delta_{k}^{*}(\mathbf{L})$.

(3) $F \in \Gamma_{k}(\mathbf{L})$.

(4) $F=\uparrow x$ for some $x \in \mathrm{D}_{k}(\mathbf{L})$.

Proof. That (1) implies (2) is immediate, and that (2) implies (3) and (3) implies (4) follow from Lemmas 3.13 and $3.11(2)$, respectively.

To see that (4) implies (1), let $x \in \mathrm{D}_{0}(\mathbf{L})$ and assume $\uparrow x \leq P \vee Q$, i.e., $\uparrow x \supseteq P \cap Q$. Suppose $P \nsubseteq \uparrow x$ and $Q \nsubseteq \uparrow x$. Choose $p \in P-\uparrow x$ and $q \in Q-\uparrow x$. Then $p \vee q \in P \cap Q$ so $p \vee q \geq x$, contradicting $x \in \mathrm{D}_{0}(\mathbf{L})$. Hence $\uparrow x \in \Delta_{0}(\mathbf{L})$.

Now let $x \in \mathrm{D}_{k}(\mathbf{L})$ with $k>0$. Assume that $\uparrow x \leq \bigvee_{i<n} P_{i}$ is a nontrivial join cover in $\mathcal{F}(\mathbf{L})$, so that $\uparrow x \supseteq \bigcap_{i<n} P_{i}$. Let the minimal nontrivial join covers of $x$ in $\mathbf{L}$ be $C_{0}, \ldots, C_{\ell-1}$. As $\mathbf{L}$ is finitely generated, there are finitely many of these, and by induction $\uparrow y \in \Delta_{k-1}(\mathbf{L})$ whenever $y \in C_{j}$. We claim that, for some $j$, $\left\{\uparrow y: y \in C_{j}\right\} \ll\left\{P_{i}: i<n\right\}$. Suppose not. Then for each $j<\ell$ there exists $c_{j} \in C_{j}$ 
such that $\uparrow c_{j} \not \leq P_{i}$, i.e., $\uparrow c_{j} \nsupseteq P_{i}$, for all $i<n$. Thus for each pair $i, j$ we can pick $p_{i j} \in P_{i}-\uparrow c_{j}$. Let $p_{i}=\bigwedge_{j<\ell} p_{i j}$. Then $\bigvee_{i<n} p_{i} \in \bigcap_{i<n} P_{i}$, so $x \leq \bigvee_{i<n} p_{i}$, but this has no refinement to one of the minimal join covers $C_{j}$. This contradiction validates the claim.

Now we must do a bit of serious work to show that $\Gamma_{k}(\mathbf{L}) \subseteq \Delta_{k}^{*}(\mathbf{L})$. This is a consequence of the following lemma.

Lemma 3.15. Let $\mathbf{L}$ be a lattice and $k>0$. Assume that $F \in \Gamma_{k}(\mathbf{L}), F \not \leq G$, $z \notin F$, and $F \leq \uparrow z \vee G$. Then there exists a filter $H$ such that $z \notin H, H \leq G$, and $H \in \Gamma_{k-1}(\mathbf{L})$.

To see that Lemma 3.15 suffices for $\Gamma_{k}(\mathbf{L}) \subseteq \Delta_{k}^{*}(\mathbf{L})$, assume that $F \in \Gamma_{k}(\mathbf{L})$ with $k>0$ and $F \leq \bigvee_{i=1}^{n} G_{i}$ nontrivially in $\mathcal{F}(\mathbf{L})$. Consider an element $z_{0} \notin F$. If $\uparrow z_{0} \vee G_{1} \geq F$, let $z=z_{0}$. If not, pick an element $z_{1} \in \uparrow z_{0} \vee G_{1}-F$, so that $z_{1} \geq z_{0}$ and $\uparrow z_{1} \geq G_{1}$. If $\uparrow z_{1} \vee G_{2} \geq F$, let $z=z_{1}$. Otherwise, continue, until we eventually get $z=z_{\ell}$ with $\ell<n$ such that $z_{\ell} \geq z_{0}$ and $\uparrow z_{\ell} \vee G_{\ell+1} \geq F$ nontrivially. Apply Lemma 3.15 to obtain $H \in \Gamma_{k-1}(\mathbf{L})$ with $H \leq G_{\ell+1}$ and $z_{\ell} \notin H$, which implies $z_{0} \notin H$. The collection of all such $H$ obtained in this way contains no $z_{0} \notin F$, so that $\bigcap H_{j} \subseteq F$, as desired. Hence $F \in \Delta_{k}^{*}(\mathbf{L})$.

To prove Lemma 3.15, we will apply the Compactness Theorem, that a set of firstorder formulas is satisfiable if every finite subset is satisfiable. It behooves us to set up the application clearly. Our language will include a set of variables $\hat{L}=\{\hat{x}: x \in \mathbf{L}\}$ corresponding to the elements of $\mathbf{L}$. It will also include logical connectives; the lattice theoretic symbols $\vee, \wedge$, and $\leq$; and two unary predicates $g$ and $h$.

Let $\Sigma$ denote the collection of formulas described as follows.

(1) $\hat{c} \neq \hat{d} \quad$ for all pairs $c \neq d$ in $\mathbf{L}$.

(2) $\hat{c} \vee \hat{d}=\hat{e} \quad$ whenever $c \vee d=e$ in $\mathbf{L}$.

(3) $\hat{c} \wedge \hat{d}=\hat{e} \quad$ whenever $c \wedge d=e$ in $\mathbf{L}$.

(4) $g(\hat{c}) \quad$ for all $c \in G$.

(5) $\neg g(\hat{d}) \quad$ for all $d \notin G$.

(6) $g(\hat{c}) \Longrightarrow h(\hat{c}) \quad$ for all $c \in \mathbf{L}$.

(7) $h(\hat{c}) \& h(\hat{d}) \Longrightarrow h(\hat{e}) \quad$ whenever $c \wedge d=e$ in $\mathbf{L}$.

(8) $h(\hat{c}) \Longrightarrow h(\hat{d}) \quad$ whenever $c \leq d$ in $\mathbf{L}$.

(9) $\neg h(\hat{z}) \quad$ for the particular element $z$.

(10) For all pairs $c, U$ with $U$ a finite subset of $\mathbf{L}$ and $c \in \operatorname{Sg}(U)$, the sentence

$$
\varphi(c, U): \quad h(\hat{c}) \Longrightarrow \text { OR }\left\{h(\hat{d}): d \leq c \text { and } d \in \mathrm{D}_{k-1}(\operatorname{Sg}(U))\right\} .
$$

Lemma 3.16. If $\Sigma$ is satisfiable, then there exists a filter $H \in \Gamma_{k-1}(\mathbf{L})$ such that $H \leq G$ and $z \notin H$.

Proof. If $\Sigma$ is satisfiable, there is an assignment of the predicate $h$ that makes conditions (1)-(10) valid. We define $H=\{x \in \mathbf{L}: h(\hat{x})\}$ and verify the conclusions of the 
lemma. In particular, $H \in \Gamma_{k-1}(\mathbf{L})$ because for each pair $c, U$ with $c \in \operatorname{Sg}(U)$, the filter $H$ satisfies the sentences $\varphi(c, U)$.

To use the Compactness Theorem, we need to show that $\Sigma$ is finitely satisfiable, i.e., to build a model for every finite subset $T \subseteq \Sigma$.

We will call a finite subset $V \subseteq \mathbf{L}$ a partial model for $T$ if it satisfies the following.

i. If the variable $\hat{x}$ appears in a formula in $T$ of type (1)-(8), then $x \in V$.

ii. $z \in V$.

iii. If $\varphi(c, U)$ is in $T$, then $c \in V, U \subseteq V$, and $\mathrm{D}_{k-1}(\operatorname{Sg}(U)) \subseteq V$.

iv. $V \cap G \neq \emptyset$.

v. $V$ is meet-closed.

Note that we have freedom in choosing an element (or elements) in $V \cap G$ required by (iv), and that because of (iv) and (v) the set $V \cap G$ has a least element $g_{V}$.

Evidently, in every partial model $V$ of $T$ the formulas of type (1)-(5) from $T$ hold with the assignment $g(\hat{x})$ if and only if $x \in G$. Thus, we need to find partial models of $T$ for which there exists an assignment of the predicate $h$ such that the formulas of type (6)-(10) hold as well.

Lemma 3.17. If there exists a partial model $V_{T}$ of $T$ such that $\mathrm{D}_{k-1}\left(\operatorname{Sg}\left(V_{T}\right)\right)$ possesses an element $a_{0}$ with the properties $a_{0} \leq g_{V_{T}}$ and $a_{0} \leq z$, then $V_{T}$ is a model for $T$.

Proof. Indeed, for $x \in V_{T}$, define $h(\hat{x})$ to hold if and only if $x \geq a_{0}$. By definition, $g_{V_{T}}$ is the least element of $V_{T} \cap G$, whence (6) holds. Properties (7)-(9) are easily checked.

That leaves (10). Consider a sentence $\varphi\left(c_{0}, U_{0}\right)$ in $T$. If $h\left(c_{0}\right)$ holds, then $a_{0} \leq c_{0}$. Now $\operatorname{Sg}\left(U_{0}\right) \leq \operatorname{Sg}\left(V_{T}\right)$, so we can apply Lemma 3.7 to obtain $\sigma\left(a_{0}\right) \leq c_{0}$ and $\sigma\left(a_{0}\right) \in$ $\mathrm{D}_{k-1}\left(\operatorname{Sg}\left(U_{0}\right)\right)$. Thus $\sigma\left(a_{0}\right) \in V_{T}$ by (iii), and since $a_{0} \leq \sigma\left(a_{0}\right)$ we have $h\left(\widehat{\sigma\left(a_{0}\right)}\right)$, so that $\varphi\left(c_{0}, U_{0}\right)$ holds.

It remains to show that under conditions of Lemma 3.15 the hypotheses of Lemma 3.17 are true.

Lemma 3.18. Let $\mathbf{L}$ be a lattice and $k>0$. Assume that $G \in \mathcal{F}(\mathbf{L})$ and for some $F \in \Gamma_{k}(\mathbf{L})$ we have $F \not \leq G, z \notin F$, and $F \leq \uparrow z \vee G$. (Note that this implies $z \notin G$ as well.) Form $\Sigma$ as above. Then for every finite subset $T \subseteq \Sigma$ there exists a partial model $V_{T}$ satisfying conditions of Lemma 3.17.

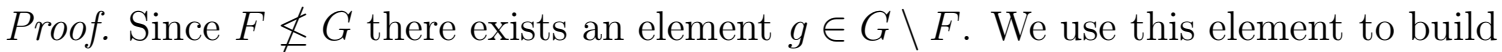
a partial model $V_{T}$ for $T$ with $g \in V_{T}$. This will imply $g_{V_{T}} \notin F$. Now $z \vee g_{V_{T}} \in \uparrow z \vee G$, and hence it is in $F$. As $F \in \Gamma_{k}(\mathbf{L})$, there exists an element $s \leq z \vee g_{V_{T}}$ with $s \in F \cap \mathrm{D}_{k}\left(\operatorname{Sg}\left(V_{I}\right)\right)$. Moreover, there exists $A \subseteq \mathrm{D}_{k-1}\left(\operatorname{Sg}\left(V_{T}\right)\right)$ with $A \ll\left\{z, g_{V_{T}}\right\}$ and $s \leq \bigvee A$. Since $z \notin F$, there is an element $a_{0} \in A$ such that $a_{0} \not \leq z$, whence $a_{0} \leq g_{V_{T}}$. 
The combination of Lemmas 3.16, 3.17 and 3.18 proves Lemma 3.15. Hence the inclusion $\Gamma_{k}(\mathbf{L}) \subseteq \Delta_{k}^{*}(\mathbf{L})$, and in view of Lemma 3.13, equality.

Theorem 3.19. For any lattice $\mathbf{L}$ and any $k \geq 0$ we have $\Delta_{k}^{*}(\mathbf{L})=\Gamma_{k}(\mathbf{L})$.

Having achieved this goal, we can drop the double notation, and henceforth refer to these sets as $\Delta_{k}^{*}(\mathbf{L})$.

3.4. Reflections into $\mathcal{L} \mathcal{B}(k)$. Now we are in a position to provide a proper generalization of Theorem 3.1. For each $k \geq 0$, there is a natural map

$$
\delta_{k}^{*}: \mathbf{L} \rightarrow\left(\Delta_{k}^{*}(\mathbf{L})\right)^{\vee} \quad \text { via } \quad \delta_{k}^{*}(x)=\bigcap\left\{F \in \Delta_{k}^{*}(\mathbf{L}): x \in F\right\} .
$$

Our main result is that the image $\delta_{k}^{*}(\mathbf{L})$ is the reflection of $\mathbf{L}$ into $\mathcal{L} \mathcal{B}(k)$.

Theorem 3.20. The following are true for any lattice $\mathbf{L}$.

(1) $\left(\Delta_{k}^{*}(\mathbf{L})\right) \bigvee \in \mathcal{L} \mathcal{B}(k)$.

(2) The map $\delta_{k}^{*}: \mathbf{L} \rightarrow\left(\Delta_{k}^{*}(\mathbf{L})\right)^{\vee}$ is a homomorphism (whence $\lambda_{k} \leq \operatorname{ker} \delta_{k}^{*}$ ).

(3) If $h: \mathbf{L} \rightarrow \mathbf{K} \in \mathcal{L} \mathcal{B}(k)$ and $h(x) \not \leq h(y)$, then there exists $P \in \Delta_{k}^{*}(\mathbf{L})$ with $x \in P$ and $y \notin P$ (whence $\left.\operatorname{ker} \delta_{k}^{*} \leq \lambda_{k}\right)$.

Hence $\lambda_{k}=\operatorname{ker} \delta_{k}^{*}$.

The rest of this section is devoted to proving this result. Part (1) is Lemma 3.21, Part (2) is Lemma 3.24, using Lemma 3.23, and Part (3) is Corollary 3.29.

Lemma 3.21. For any lattice $\mathbf{L},\left(\Delta_{k}^{*}(\mathbf{L})\right)^{\vee} \in \mathcal{L} \mathcal{B}(k)$.

Proof. Put $\mathbf{M}=\left(\Delta_{k}^{*}(\mathbf{L})\right)^{\vee}$. A straightforward induction shows that $\Delta_{j}^{*}(\mathbf{L}) \subseteq \mathrm{D}_{j}^{*}(\mathbf{M})$ for $j \leq k$. Hence $\left(\mathrm{D}_{k}^{*}(\mathbf{M})\right)^{\vee}=\mathbf{M}$. By Corollary 3.9, $\mathbf{M} \in \mathcal{L} \mathcal{B}(k)$.

Next, we investigate when natural maps are homomorphisms, generalizing slightly an old result for finite lattices ([5], see also section III.2 of [4]).

Definition 3.22. Let $\mathbf{L}$ be a lattice and $\mathcal{U} \subseteq \mathcal{F}(\mathbf{L})$. We say that $\mathcal{U}$ has the weak refinement property (WRP) if whenever $x \vee y \in G \in \mathcal{U}$, then there exist (not necessarily finite) collections $H_{i}(i \in I)$ and $K_{j}(j \in J)$ such that

(1) $x \in H_{i} \in \mathcal{U}$ for all $i$

(2) $y \in K_{j} \in \mathcal{U}$ for all $j$,

(3) $G \leq \bigvee_{i \in I} H_{i} \vee \bigvee_{j \in J} K_{j}$, i.e., $G \supseteq \bigcap_{i \in I} H_{i} \cap \bigcap_{j \in J} K_{j}$.

Lemma 3.23. If $\mathcal{U}$ has the WRP, then the natural map $\varphi: \mathbf{L} \rightarrow \mathcal{U}^{\vee}$ via $\varphi(x)=$ $\bigcap\{F \in \mathcal{U}: x \in F\}$ is a homomorphism.

Proof. The operations in $\mathcal{U}$ are given by

$$
\begin{aligned}
& X \vee Y=X \cap Y \\
& X \wedge Y=\bigcap\{F \in \mathcal{U}: X \cup Y \subseteq F\} .
\end{aligned}
$$


In order to show that $\varphi(x \vee y) \leq \varphi(x) \vee \varphi(y)$, we need to show that if $a \in \varphi(x) \cap \varphi(y)$, then $x \vee y \in G \in \mathcal{U}$ implies that $a \in G$. Assume that $a \in \varphi(x)$, so that $x \in F \in \mathcal{U}$ implies $a \in F$, and also $a \in \varphi(y)$, interpreted similarly. Suppose $x \vee y \in G \in \mathcal{U}$ (i.e., $\uparrow x \vee \uparrow y \geq G)$. By the WRP we get collections of filters $H_{i}(i \in I)$ and $K_{j}$ $(j \in J)$ such that $x \in H_{i} \in \mathcal{U}$ for all $i$, and similarly $y \in K_{j} \in \mathcal{U}$ for all $j$, and $G \supseteq \bigcap H_{i} \cap \bigcap K_{j}$. Then $a \in \varphi(x)$ implies that $a \in H_{i}$ for all $i$, and likewise $a \in K_{j}$ for all $j$. Hence $a \in G$, as desired.

To show that $\varphi(x) \wedge \varphi(y) \leq \varphi(x \wedge y)$, let $a \in \varphi(x \wedge y)$, so that $x \wedge y \in F \in \mathcal{U}$ implies $a \in F$. Suppose that $\varphi(x) \cup \varphi(y) \subseteq G \in \mathcal{U}$. Then, in particular, $x$ and $y$ are both in $G$, whence $x \wedge y \in G$. Thus $a \in G$, as was to be shown.

It is clear from the definitions that $\Delta_{k}^{*}(\mathbf{L})$ has the WRP, so by Lemma 3.23 we have one more of our desired conclusions.

Lemma 3.24. $\delta_{k}^{*}$ is a homomorphism.

That leaves part (3) of Theorem 3.20 to be proved.

Lemma 3.25. Let $\mathbf{L} \in \mathcal{L} \mathcal{B}(k)$. If $G \in \mathcal{F}(\mathbf{L})$ and $z \in \mathbf{L}$ with $z \notin G$, then there exists $H \in \Delta_{k}^{*}(\mathbf{L})$ such that $H \leq G$ and $z \notin H$.

Proof. Build the set of first order sentences $\Sigma$ used in the proof of Lemma 3.15, except that in $(10) k-1$ is replaced by $k$, so that $\varphi(c, U)$ reads

$$
h(\hat{c}) \Longrightarrow \operatorname{OR}\left\{h(\hat{d}): d \leq c \text { and } d \in \mathrm{D}_{k}(\operatorname{Sg}(U))\right\} .
$$

According to Lemma 3.16, again with $k-1$ replaced by $k$, it is enough to prove that $\Sigma$ is satisfiable. Hence, we need to check that it is finitely satisfiable. For this we want to verify that if $\mathbf{L} \in \mathcal{L B}(k)$ then the hypotheses of Lemma 3.17, with $k-1$ replaced by $k$, are true.

Given a finite subset $T \subseteq \Sigma$, we take any partial model $V$ of $T$ and define $V_{T}=$ $\operatorname{Sg}(V)$. Since $\mathbf{L} \in \mathcal{L} \mathcal{B}(k)$ and $\mathcal{L} \mathcal{B}(k)$ is locally finite, $V_{T}$ is again a partial model for $T$. As before, $g_{V_{T}}$ denotes the least element in $V_{T} \cap G$.

Since $g_{V_{T}} \not \leq z$ and $V_{T} \in \mathcal{L} \mathcal{B}(k)$, there is an element $a_{0} \in \mathrm{D}_{k}\left(\operatorname{Sg}\left(V_{T}\right)\right)=\mathrm{D}_{k}\left(V_{T}\right)$ with $a_{0} \leq g_{V_{T}}$ and $a_{0} \not \leq z$. Hence, according to Lemma 3.17, $V_{T}$ is a model for $T$.

Corollary 3.26. Let $\mathbf{L} \in \mathcal{L} \mathcal{B}(k)$. If $x \not \leq y$, then there exists $H \in \Delta_{k}^{*}(\mathbf{L})$ such that $x \in H$ and $y \notin H$.

Proof. Let $F=\uparrow x$ and apply Lemma 3.25.

The next lemma is straightforward.

Lemma 3.27. Let $\mathbf{A}$ and $\mathbf{B}$ be lattices with $\mathbf{A}$ finitely generated and $\mathbf{B}$ lower bounded. Let $h: \mathbf{A} \rightarrow \mathbf{B}$ be a homomorphism, and let $\beta_{h}$ be the corresponding lower bound map. If $a \in \mathrm{D}_{k}(\mathbf{B})$, then $\beta_{h}(a) \in \mathrm{D}_{k}(\mathbf{A})$. 
Lemma 3.28. If $h: \mathbf{L} \rightarrow \mathbf{K} \in \mathcal{L} \mathcal{B}(k)$ and $F \in \Delta_{k}^{*}(\mathbf{K})$, then $h^{-1}(F) \in \Delta_{k}^{*}(\mathbf{L})$.

Proof. W.l.o.g. we may assume that $h$ is surjective. Let $x \in h^{-1}(F)$ and suppose $U$ is a finite set with $x \in \operatorname{Sg}(U)$. Then $h(x) \in \operatorname{Sg}(h(U)) \cap F$, so there exists $y \leq h(x)$ with $y \in \mathrm{D}_{k}(\operatorname{Sg}(h(U))) \cap F$. Using the $\operatorname{map} \beta$ for $\left.h\right|_{\operatorname{Sg}(U)}$ we have

$$
\begin{aligned}
& \beta(y) \leq \beta(h(x)) \leq x \\
& \beta(y) \in h^{-1}(F) \\
& \beta(y) \in \mathrm{D}_{k}(\operatorname{Sg}(U))
\end{aligned}
$$

with the last claim using the preceding lemma. Hence $h^{-1}(F) \in \Delta_{k}^{*}(\mathbf{L})$.

Combining Corollary 3.26 and Lemma 3.28, we obtain the following.

Corollary 3.29. Let $h: \mathbf{L} \rightarrow \mathbf{K}$ with $\mathbf{K} \in \mathcal{L} \mathcal{B}(k)$. If $h(x) \not \leq h(y)$, then there exists $G \in \Delta_{k}^{*}(\mathbf{L})$ such that $x \in G$ and $y \notin G$.

This completes the proof of Theorem 3.20. As a consequence, we have two characterizations of the lattices in $\mathcal{L} \mathcal{B}(k)$. For a complete lattice $\mathbf{K}$, let $\mathrm{CJ}(\mathbf{K})$ denote the set of completely join irreducible elements of $\mathbf{K}$.

Corollary 3.30. $\mathbf{L} \in \mathcal{L} \mathcal{B}(k)$ if and only if, for every pair $x \not \leq y$ in $\mathbf{L}$, there exists $H \in \Delta_{k}^{*}(\mathbf{L})$ such that $x \in H$ and $y \notin H$.

This is just a translation of the condition $\lambda_{k}=0$.

Corollary 3.31. $\mathbf{L} \in \mathcal{L} \mathcal{B}(k)$ if and only if $\mathrm{CJ}(\mathcal{F}(\mathbf{L})) \subseteq \Delta_{k}^{*}(\mathbf{L})=\mathrm{D}_{k}^{*}(\mathcal{F}(\mathbf{L}))$.

Proof. Assume $\mathbf{L} \in \mathcal{L} \mathcal{B}(k)$. By Lemma 3.25, every filter of $\mathbf{L}$ is the join (set intersection) of filters in $\Delta_{k}^{*}(\mathbf{L})$, and so $\mathrm{CJ}(\mathcal{F}(\mathbf{L})) \subseteq \Delta_{k}^{*}(\mathbf{L})$.

Conversely, assume $\mathrm{CJ}(\mathcal{F}(\mathbf{L})) \subseteq \Delta_{k}^{*}(\mathbf{L})$. In a dually algebraic lattice, every element is the join of completely join irreducible elements. Corollary 3.9 then yields that $\mathcal{F}(\mathbf{L}) \in \mathcal{L} \mathcal{B}(k)$, whence also $\mathbf{L} \in \mathcal{L} \mathcal{B}(k)$.

Corollary 3.31 is the correct generalization of the statement that a finitely generated lattice $\mathbf{L}$ is in $\mathcal{L} \mathcal{B}(k)$ if and only if $\mathrm{J}(\mathbf{L}) \subseteq \mathrm{D}_{k}(\mathbf{L})$. For the non-finitely-generated case, we must use $\mathrm{D}_{k}^{*}$ and pass to the filter lattice.

\section{Some observations about lower Bounded LatTices}

There are not many facts discovered so far about the class $\mathcal{L} \mathcal{B}$ of lower bounded lattices. We collect what is known in the following statement. Item (2) was kindly offered by M. Semenova.

Proposition 4.1. (1) $\mathcal{L} \mathcal{B}$ is closed under taking sublattices.

(2) $\mathcal{L} \mathcal{B}$ is closed under lower bounded homomorphisms.

(3) $\mathcal{L} \mathcal{B}$ is not closed under taking direct products. 
(4) Every lower bounded lattice is embedded into an ultraproduct of finite lower bounded lattices.

Proof. (1) is evident from the definition of $\mathcal{L} \mathcal{B}$. (Note however that the definition is motivated by the nontrivial fact that if $\mathbf{L}$ is a lower bounded homomorphic image of a free lattice and $\mathbf{K}$ is a finitely generated sublattice of $\mathbf{L}$, then $\mathbf{K}$ is a lower bounded homomorphic image of a free lattice. One proof of this is to apply Lemma 3.7 above.)

(2) Indeed, assume $\mathbf{L}_{1} \in \mathcal{L} \mathcal{B}$, and let $f: \mathbf{L}_{1} \rightarrow \mathbf{L}_{2}$ be a lower bounded epimorphism. Let $g: \mathbf{F L}(n) \rightarrow \mathbf{L}_{2}$ be a homomorphism from a finitely generated free lattice. We want to show that $g$ is a lower bounded homomorphism. Since $\mathbf{F L}(n)$ is projective and $f$ is surjective, there is a homomorphism $h: \mathbf{F L}(n) \rightarrow \mathbf{L}_{1}$ such that $g=f h$. The map $h$ is lower bounded as $\mathbf{L}_{1} \in \mathcal{L} \mathcal{B}$. Because $f$ and $h$ are lower bounded homomorphisms, there are lower bound (partial) maps $\beta_{f}: \mathbf{L}_{2} \rightarrow \mathbf{L}_{1}$ and $\beta_{h}: \mathbf{L}_{1} \rightarrow \mathbf{F L}(n)$. It is straightforward to check that since $g=f h$, the composition $\beta_{h} \circ \beta_{f}$ is a lower bound map for $g$.

(3) follows from item 3 in the proof of Theorem 1.1 in [2]. In particular, $\mathbf{L}=$ $\Pi_{k \in \omega} \operatorname{Sub}_{\wedge}(B(k))$ is not lower bounded. Here $B(k)$ is a Boolean lattice with $k$ atoms, and $\operatorname{Sub}_{\wedge}(\mathbf{P})$ denotes the lattice of subsemilattices of any $\wedge$-semilattice $\mathbf{P}$.

(4) is proved in [2].

Question 4.2. Is $\mathcal{L} \mathcal{B}$ closed under ultraproducts?

At the moment we do not know any characterization of lower bounded lattices in the general case. An interesting class was treated in Semenova [12].

Theorem 4.3. A lattice of suborders is lower bounded iff it satisfies the weak Jónsson property.

Another class of (not necessarily finitely generated) lower bounded lattices was given in Adaricheva and Gorbunov [2]. These are the lattices $\operatorname{Sub}_{\mathrm{f}}(\mathbf{P})$ of finite subsemilattices of a semilattice $\mathbf{P}$. The weak Jónsson property may fail there, but these lattices are locally finite.

The main goal of this section is to give another sufficient condition for a lattice to be lower bounded (Theorem 4.4). This allows us to build a new example of a (non-finitely-generated) lower bounded lattice that is not locally finite and that does not satisfy the weak Jónsson property.

Theorem 4.4. Let $\mathbf{L}$ be a lattice additively generated by a set A, i.e., every element of $\mathbf{L}$ is a (possibly infinite) join of elements from $A$. If $A \subseteq \mathrm{D}^{*}(\mathbf{L})$ and every finitely generated sublattice of $\mathbf{L}$ satisfies the ascending chain condition, then $\mathbf{L}$ is lower bounded.

Proof. It is enough to show that $\mathrm{D}(\mathbf{S})=\mathbf{S}$ for any finitely generated sublattice $\mathbf{S}$ of L. 
For $a \in A$ such that $\uparrow a \cap \mathbf{S}$ is nonempty, let $\sigma(a)$ be the least element of $\uparrow a \cap \mathbf{S}$. According to Lemma 3.7, such an element always exists as $a \in \mathrm{D}^{*}(\mathbf{L})$.

For any $t \in \mathbf{S}$, let $A_{t}=\{a \in A: a \leq t\}$. We claim that $t=\bigvee_{a \in A_{t}} \sigma(a)$. Evidently, $t=\bigvee A_{t}$, since $A$ additively generates $\mathbf{L}$ and $t \geq \sigma(a) \geq a$ for any $a \in A_{t}$, whence the claim follows. (Note that we do not require $\mathbf{L}$ to be a complete lattice!)

Now we want to show that, in fact, there exist finitely many elements $a_{0}, \ldots, a_{k} \in$ $A_{t}$ such that $t=\sigma\left(a_{0}\right) \vee \ldots \vee \sigma\left(a_{k}\right)$. Consider the set $A^{\prime}=\left(\sigma(a): a \in A_{t}\right)^{\vee}$, and note that $A^{\prime} \subseteq \mathrm{S}$. If $t \notin A^{\prime}$, then $A^{\prime}$ contains infinite increasing chain that contradicts the ascending chain condition in $\mathbf{S}$. Hence $t \in A^{\prime}$.

Since $A \subseteq \mathrm{D}^{*}(\mathbf{L})$, we have $a_{0}, \ldots, a_{k} \in \mathrm{D}_{p}^{*}(\mathbf{L})$ for some $p<\omega$. From Lemma 3.7 it follows that $\sigma\left(a_{0}\right), \ldots, \sigma\left(a_{k}\right) \in \mathrm{D}_{p}(\mathbf{S})$. Hence $t \in \mathrm{D}_{p+1}(\mathbf{S})$ and $\mathbf{S} \subseteq \mathrm{D}(\mathbf{L})$.

Note that in Theorem 4.4 we cannot drop the requirement that every finitely generated sublattice of $\mathbf{L}$ satisfies the ACC. Indeed, the lattice $\mathbf{L}=\mathrm{O}(\omega)$ of suborders of $\omega$ is not lower bounded [13]. On the other hand, it is additively generated by the set of atoms $A$, for which $A \subseteq \mathrm{D}(\mathbf{L}) \subseteq \mathrm{D}^{*}(\mathbf{L})$ evidently holds.

We want to start the description of a new example of a lower bounded lattice.

Let $\mathbf{P}_{1}$ be a $\wedge$-semilattice that consists of two infinite decreasing chains $A=\left(a_{i}\right.$ : $i \in \omega)$ and $B=\left(b_{i}: i \in \omega\right)$ with the relations $a_{0}<b_{0}$ and $a_{i+1}=b_{i+1} \wedge a_{i}$ for all $i \geq 0$. (See Figure 1.) Thus $\mathbf{P}_{1} \cong \omega^{d} \times \mathbf{2}$, where $\omega^{d}$ denotes the dual of $\omega$. Let $\mathbf{L}_{1}=\operatorname{Sub}\left(\mathbf{P}_{1}\right)$ be the lattice of subsemilattices of $\mathbf{P}_{1}$.

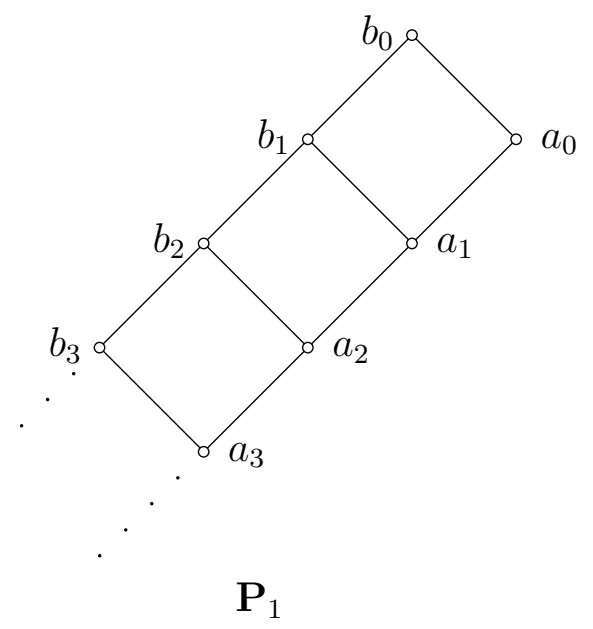

FiguRE 1

The following series of lemmas describes different features of $\mathbf{L}_{1}$.

Lemma 4.5. $\mathbf{L}_{1}$ is an atomistic lattice, and the set of atoms $\operatorname{At}\left(\mathbf{L}_{1}\right)$ satisfies $\operatorname{At}\left(\mathbf{L}_{1}\right) \subseteq$ $\mathrm{D}^{*}\left(\mathbf{L}_{1}\right)$. 
Proof. Indeed, the atoms of $\mathbf{L}_{1}$ are the one-element subsets of $\mathbf{P}_{1}$. The subsemilattices $\left\{a_{0}\right\}$ and $\left\{b_{k}\right\}$ for $k \geq 0$ are join prime, as the corresponding elements of $\mathbf{P}_{1}$ are meet irreducible. Using the fact that $\left\{a_{k+1}\right\} \leq\left\{b_{k+1}\right\} \vee\left\{a_{k}\right\}$ in $\mathbf{L}_{1}$, one can verify inductively that $\mathrm{D}_{k}\left(\mathbf{L}_{1}\right) \cap \operatorname{At}\left(\mathbf{L}_{1}\right)=\left\{a_{0}, \ldots, a_{k}\right\} \cup B$ for all $k \geq 0$.

Lemma 4.6. $\mathbf{L}_{1}$ does not satisfy the weak Jónsson property.

Proof. Indeed, consider $A=\left\{a_{i}: i \in \omega\right\} \in \mathbf{L}_{1}$. Evidently, $A_{i}=A \backslash\left\{a_{i}\right\}$ is an element of $\mathbf{L}_{1}$ for any $i \in \omega$. Now $A \leq\left\{a_{i}\right\} \vee A_{i}$ is a nontrivial join cover of $A$, so $A \notin \mathrm{D}_{0}^{*}\left(\mathbf{L}_{1}\right)$. Moreover, since $\left\{a_{i}\right\}$ is an atom of $\mathbf{L}_{1}$ and $A \not \leq A_{i},\left\{a_{i}\right\}$ will be present in any refinement of this join cover. For $i>0$ we note that $\left\{a_{i}\right\} \notin \mathrm{D}_{i-1}^{*}\left(\mathbf{L}_{1}\right)$ by the proof of Lemma 4.5 , whence $A \notin \mathrm{D}_{i}^{*}\left(\mathbf{L}_{1}\right)$. Thus $A \notin \bigcup_{i \geq 0} \mathrm{D}_{i}^{*}\left(\mathbf{L}_{1}\right)=\mathrm{D}^{*}\left(\mathbf{L}_{1}\right)$.

Lemma 4.7. Every finitely generated sublattice of $\mathbf{L}_{1}$ satisfies the ascending chain condition.

Proof. For any subset $t \subseteq \mathbf{P}_{1}$, let $t^{A}=\left\{i \in \omega: a_{i} \in t\right\}$ and $t^{B}=\left\{i \in \omega: b_{i} \in t\right\}$.

Claim 1. The subset $t$ is in $\operatorname{Sub}\left(\mathbf{P}_{1}\right)$ if and only if either $t^{A}$ is empty, or $t^{A}$ is nonempty and the integer $i_{0}=\bigwedge t^{A}$ satisfies $\uparrow i_{0} \cap t^{B} \subseteq t^{A}$.

The condition is equivalent to saying that $t^{B}$ is arbitrary, and if $a_{i} \in t, b_{j} \in t$ and $j>i$, then $a_{j} \in t$. As a consequence, we can describe the join in $\mathbf{L}_{1}$.

Claim 2. For any $s, q \in \operatorname{Sub}\left(\mathbf{P}_{1}\right)$, the following hold.

(1) $(s \wedge q)^{A}=s^{A} \cap q^{A}$ and $(s \wedge q)^{B}=s^{B} \cap q^{B}$.

(2) If $s^{A} \cup q^{A} \neq \emptyset$ and $m_{s, q}=\bigwedge\left(s^{A} \cup q^{A}\right)$, then $(s \vee q)^{A}=s^{A} \cup q^{A} \cup\left(\uparrow m_{s, q} \cap\right.$ $\left.\left(s^{B} \cup q^{B}\right)\right)$, and $(s \vee q)^{B}=s^{B} \cup q^{B}$.

Consider a sublattice $\mathbf{S}$ of $\mathbf{L}_{1}$ generated by elements $X_{1}, \ldots, X_{k}$. Let $\mathcal{I}_{X}=$ $\left\{X_{1}^{A}, \ldots, X_{k}^{A}, X_{1}^{B}, \ldots, X_{k}^{B}\right\}$ and let $I=\bigcup_{i \leq k} X_{i}^{A} \cup \bigcup_{i \leq k} X_{i}^{B}$. The next two claims are consequences of Claim 2.

Claim 3. Let $\mathcal{B}=\left\{X_{1}^{B}, \ldots, X_{k}^{B}\right\}^{\wedge \vee}$, where $\wedge, \vee$ are the operations of intersection and union of sets in the lattice $B(\omega)$. Then for any $t \in \mathbf{S}$ we have $t^{B} \in \mathcal{B}$.

Claim 4. If $t \in \mathbf{S}$ then $t^{A} \cup t^{B} \subseteq I$.

For any $i \in \omega$ such that $i \in t^{A}$ for some $t \in \mathbf{S}$, we define two sets: $N^{i}=\bigcap\{Y \in$ $\left.\mathcal{I}_{X}: i \in Y\right\}$ and $M^{i}=N^{i} \cap \uparrow i$. Thus $N^{i}=\left\{j \in \omega: i \in Y \in \mathcal{I}_{X} \Longrightarrow j \in Y\right\}$ and $M^{i}=\left\{j \in \omega: j \geq i\right.$ and $\left.i \in Y \in \mathcal{I}_{X} \Longrightarrow j \in Y\right\}$. According to Claim 4, there is at least one $Y \in \mathcal{I}_{X}$ such that $i \in Y$, whence $N^{i}$ is well-defined and $i \in N^{i}$. The next statement complements Claim 4.

Claim 5. If $t \in \mathbf{S}$ then $M^{i} \subseteq t^{A} \cap t^{B}$. 
Proof. That $M^{i} \subseteq N^{i} \subseteq t^{B}$ is a consequence of Claim 3 .

We use induction to prove that $M^{i} \subseteq t^{A}$. If $t=X_{j}$ and $i \in X_{j}^{A}$ then, evidently, $M^{i} \subseteq X_{j}^{A}$. If $t=s \wedge q$ and $i \in t^{A}$ then $i \in s^{A} \cap q^{A}$, hence $M^{i} \subseteq t^{A}$ follows from the inductive hypothesis. Finally, if $t=s \vee q$ and $i \in t^{A}$ then, by Claim 2, we get $i \in s^{A} \cup q^{A} \cup\left(\left(s^{B} \cup q^{B}\right) \cap \uparrow m_{s, q}\right)$. If $i \in s^{A} \cup q^{A}$, then, by the inductive hypothesis, $M^{i} \subseteq s^{A} \cup q^{A} \subseteq t^{A}$. If $i \in\left(s^{B} \cup q^{B}\right) \cap \uparrow m_{s, q}$ then $i \in s^{B} \cup q^{B}$ and $i \geq m_{s, q}$. We have already noted that $M^{i} \subseteq t^{B}=s^{B} \cup q^{B}$. Also $\uparrow i \subseteq \uparrow m_{s, q}$, which implies $M^{i} \subseteq \uparrow i \subseteq \uparrow m_{s, q}$. Hence, $M^{i} \subseteq\left(s^{B} \cup q^{B}\right) \cap \uparrow m_{s, q} \subseteq t^{A}$ and we are done.

Now we are ready to prove the statement of Lemma 4.7. Suppose that there exists an infinite increasing chain $t_{1}<t_{2}<\ldots<t_{n}<\ldots$ in $\mathbf{S}$. In view of Claim 3, we may assume that $t_{n}^{B}=t_{m}^{B}$ for all $n, m \in \omega$. Hence, for any $n>1$ we can find $i_{n} \in t_{n}^{A} \backslash t_{n-1}^{A}$. Note that $i_{m} \notin t_{n}^{A}$ whenever $m>n$. Possibly reducing the sequence $\left(t_{i}, i \in \omega\right)$ to an infinite subsequence, we can always assume that $i_{n+1}>i_{n}$.

Consider the sets $N^{i_{n}}, n \in \omega$. Since every $N^{i_{n}}$ is the intersection of members of the finite family $\mathcal{I}_{X}$, there are infinitely many $i_{n}$ for which $N^{i_{n}}$ is the same set, say, $N$. Without loss of generality, we may assume that $N=N^{i_{n}}$ for all $n \in \omega$. Therefore $i_{n} \in N$ for every $n$.

Since $i_{n} \in t_{n}^{A}$, it follows from Claim 5 that $M^{i_{n}}=N \cap \uparrow i_{n} \subseteq t_{n}^{A}$. In particular, $i_{m} \in t_{n}^{A}$ for all $m>n$, a contradiction.

The direct consequence of Theorem 4.4, Lemmas 4.5 and 4.7 is

Theorem 4.8. $\operatorname{Sub}\left(\mathbf{P}_{1}\right)$ is lower bounded.

Lemma 4.9. $\mathbf{L}_{1}$ contains an infinite 3 -generated sublattice. In particular, $\mathbf{L}_{1}$ is not locally finite.

Proof. Let $\mathbf{S}$ be the sublattice of $\mathbf{L}_{1}=\operatorname{Sub}\left(\mathbf{P}_{1}\right)$ generated by the three elements described as follows.

$$
\begin{aligned}
& X_{0}^{A}=X_{0}^{B}=\{i \in \omega: i \equiv 0 \bmod 2\} \\
& X_{1}^{A}=X_{1}^{B}=\{i \in \omega: i \equiv 1 \bmod 2\} \\
& X_{2}^{A}=\emptyset \text { and } X_{2}^{B}=\uparrow 2=\{i \in \omega: i \geq 2\} .
\end{aligned}
$$

This sublattice is illustrated in Figure 2; we sketch enough of the calculations to show that $\mathbf{S}$ contains infinite descending chains. Let $Y_{0}=X_{0} \wedge X_{2}, Y_{1}=X_{1} \wedge X_{2}$, $Z_{0}=X_{0} \vee X_{2}$ and $Z_{1}=X_{1} \vee X_{2}$, so that

$$
\begin{aligned}
& Y_{0}^{A}=\emptyset \text { and } Y_{0}^{B}=\{i \in \omega: i \geq 2 \text { and } i \equiv 0 \bmod 2\} \\
& Y_{1}^{A}=\emptyset \text { and } Y_{1}^{B}=\{i \in \omega: i \geq 3 \text { and } i \equiv 1 \bmod 2\} \\
& Z_{0}^{A}=Z_{0}^{B}=\{0\} \cup \uparrow 2 \\
& Z_{1}^{A}=Z_{1}^{B}=\uparrow 1 .
\end{aligned}
$$


Then let $U_{0}=X_{0}, V_{1}=X_{1}$, and inductively define $U_{k+2}=X_{0} \wedge\left(V_{k+1} \vee X_{2}\right)$ and $V_{k+2}=X_{1} \wedge\left(U_{k+1} \vee X_{2}\right)$. The descriptions of $U_{k}$ for $k \geq 2$ even, and $V_{k}$ for $k \geq 3$ odd, along with the auxiliary elements

$$
M_{k}= \begin{cases}U_{k} \vee X_{2} & \text { if } k \geq 2 \text { even } \\ V_{k} \vee X_{2} & \text { if } k \geq 3 \text { odd }\end{cases}
$$

are given by

$U_{k}^{A}=\{i \in \omega: i \geq k$ and $i \equiv 0 \bmod 2\}$ and $U_{k}^{B}=\{i \in \omega: i \geq 2$ and $i \equiv 0 \bmod 2\}$

$V_{k}^{A}=\{i \in \omega: i \geq k$ and $i \equiv 1 \bmod 2\}$ and $V_{k}^{B}=\{i \in \omega: i \geq 3$ and $i \equiv 1 \bmod 2\}$

$M_{k}^{A}=\uparrow k$ and $M_{k}^{B}=\uparrow 2$.

These calculations are straightforward, using Claim 2 of the proof of Lemma 4.7.

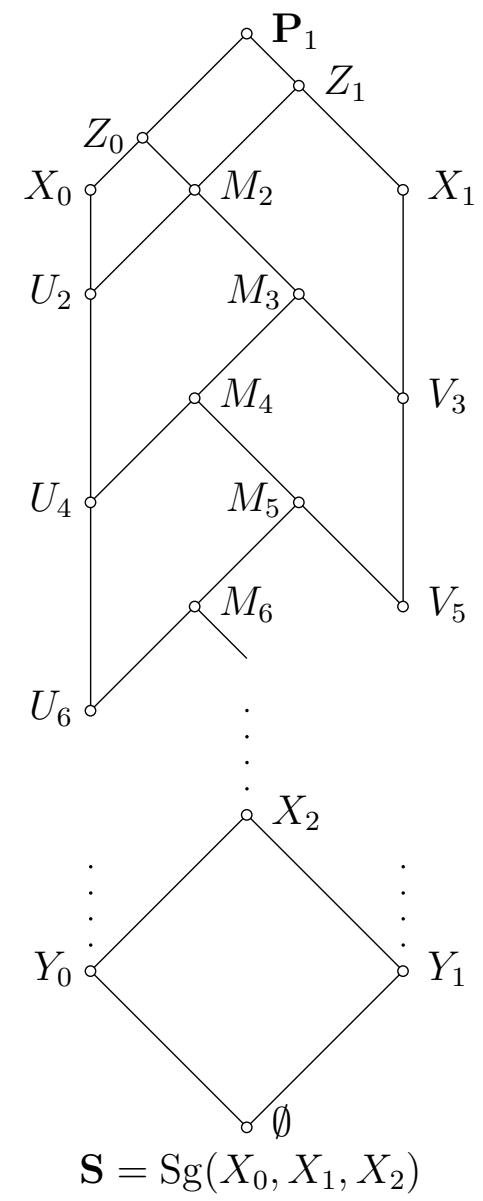

Figure 2 
Combining Lemma 4.6, Theorem 4.8 and Lemma 4.9, we see that $\mathbf{L}_{1}$ has the desired properties.

Theorem 4.10. $\operatorname{Sub}\left(\mathbf{P}_{1}\right)$ is lower bounded, is not locally finite, and does not satisfy the weak Jónsson property.

Another interesting observation about this example is that there is an overlattice $\mathbf{L}_{2}$ of $\mathbf{L}_{1}$ that has just two extra atoms and is not lower bounded.

Let $\mathbf{P}_{2}=\mathbf{P}_{1} \cup\left\{b_{\omega}, a_{\omega}\right\}$ be a semilattice extending $\mathbf{P}_{1}$ by two elements. We extend the meet operation by $a_{\omega}<b_{\omega}<b_{i}$ and $a_{\omega}=b_{\omega} \wedge a_{i}$ for all $i<\omega$. (See Figure 3). Let $\mathbf{L}_{2}=\operatorname{Sub}\left(\mathbf{P}_{2}\right)$. Of course, $\operatorname{Sub}\left(\mathbf{P}_{1}\right) \leq \operatorname{Sub}\left(\mathbf{P}_{2}\right)$. We also extend the definition of $t^{A}$ and $t^{B}$ to $\omega+1$.

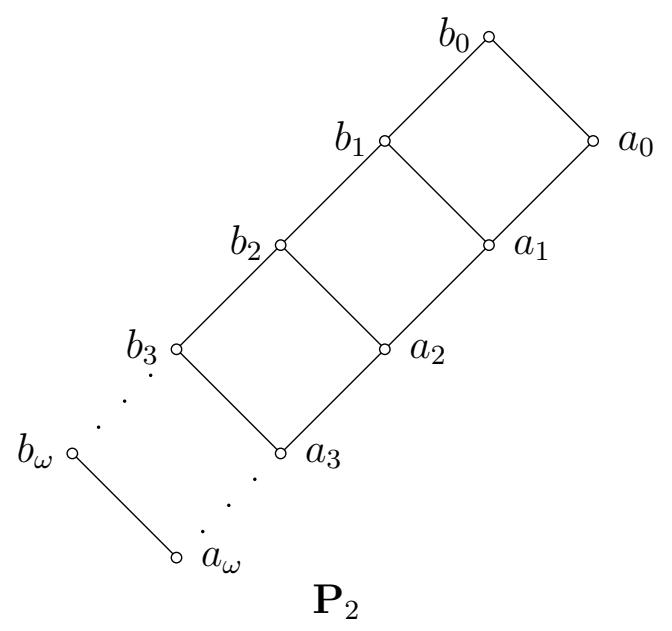

Figure 3

Theorem 4.11. $\operatorname{Sub}\left(\mathbf{P}_{2}\right)$ is not lower bounded.

Proof. Let $\mathbf{S}_{6}=\operatorname{Sg}\left(X_{0}, X_{1}, X_{2}, X_{3}, X_{4}, X_{5}\right)$ be the sublattice of $\operatorname{Sub}\left(\mathbf{P}_{2}\right)$ that is generated by $X_{0}, X_{1}, X_{2}$ of Lemma 4.9 and $X_{3}=\left\{a_{i}: i<\omega\right\}, X_{4}=\left\{b_{\omega}\right\}, X_{5}=\left\{a_{\omega}\right\}$.

Define a sequence of subsemilattices $W_{k}$ for even integers $k<\omega$ by the rule $W_{k}=$ $U_{k} \wedge X_{3}$. Then $W_{k}^{A}=\{i \in \omega: i \geq k$ and $i \equiv 0 \bmod 2\}$ and $W_{k}^{B}=\emptyset$. We want to prove that, for any nonempty $t \in \mathbf{S}_{6}$, if $t \leq W_{k}$ then $t=W_{k+s}$ for some $s \geq 0$.

For this, let's show that any $t \in \mathbf{S}_{6}$ satisfies the following properties.

(i) If $i<\omega$ and $i \in t^{A}$ then $(i+2) \in t^{A}$.

(ii) If $i<\omega$ and $i \in t^{B}$ then $(i+2) \in t^{B}$.

Note that the generators of $\mathbf{S}_{6}$ satisfy (i) and (ii). We use Claim 2 from the proof of Lemma 4.7 (extended) to show that the properties are preserved under meet and join. Assume that $s$ and $t$ satisfy (i) and (ii). Then it is clear that the property holds for $(t \wedge s)^{A},(t \wedge s)^{B}$ and $(t \vee s)^{B}$. For the remaining case, if $t^{A} \cup s^{A} \neq \emptyset$, then 
$(t \vee s)^{A}=t^{A} \cup s^{A} \cup\left(\left(t^{B} \cup s^{B}\right) \cap \uparrow m_{s, t}\right)$, where $m_{s, t}=\bigwedge\left(t^{A} \cup s^{A}\right)$. Evidently, the property holds for each term on the right hand side. If $t^{A} \cup s^{A}=\emptyset$, then $(t \vee s)^{A}=\emptyset$, and we are done.

Now assume $t \leq W_{k}$ and $t \neq \emptyset$. Let $j=\bigwedge t^{A}$. Then $j=k+s$ for some even integer $s \geq 0$. By the property (i), we have that $W_{k+s} \subseteq t$, whence $t=W_{k+s}$.

For every even integer $k \geq 0$, we get a nontrivial join cover in $\mathbf{S}_{6}: X_{5} \leq X_{4} \vee W_{k}$. This cannot be a minimal join cover since $X_{5} \leq X_{4} \vee W_{k+2}$ and $\left\{X_{4}, W_{k+2}\right\} \ll$ $\left\{X_{4}, W_{k}\right\}$. Moreover, if $\left\{t_{1}, \ldots, t_{p}\right\} \ll\left\{X_{4}, W_{k}\right\}$ and $X_{5} \leq t_{1} \vee \ldots t_{p}$, then (possibly reindexing) for some $j$ with $1 \leq j<p$ we have $t_{1}, \ldots, t_{j} \leq X_{4}$ and $t_{j+1}, \ldots, t_{p} \leq W_{k}$. Then $t_{1}=\ldots=t_{j}=X_{4}$ and $t_{q}=W_{k+s_{q}}$ for $q \in\{j+1, \ldots, p\}$ and some even integers $s_{q} \geq 0$. Thus, in $\mathbf{S}_{6}$, any nontrivial join cover of $X_{5}$ that refines $X_{4} \vee W_{k}$ refines to one of the form $X_{4} \vee W_{k+s}$. So the join cover $X_{5} \leq X_{4} \vee W_{k}$ cannot be refined to a minimal one.

By Corollary 2.19 of [4], every finitely generated lower bounded lattice has the minimal join cover refinement property (every nontrivial join cover of a fixed element refines to one of a finite set of minimal ones). Since $\mathbf{S}_{6}$ is finitely generated, we conclude that it is not lower bounded. Hence, $\operatorname{Sub}\left(\mathbf{P}_{2}\right)$ is not lower bounded as well.

Note that adding two elements $b_{\omega}, a_{\omega}$ to $\mathbf{P}_{1}$ we made the lattice of subsemilattices not lower continuous (see [1]). This was essentially used in the proof of Theorem 4.11: the minimal join cover refinement property failed for the same reason as lower continuity.

Note also that one of two properties required in Theorem 4.4 fails in $\operatorname{Sub}\left(\mathbf{P}_{2}\right)$. Namely, $\operatorname{Sub}\left(\mathbf{P}_{2}\right)$ is atomistic, but $\operatorname{At}\left(\operatorname{Sub}\left(\mathbf{P}_{2}\right)\right) \nsubseteq \mathrm{D}^{*}\left(\operatorname{Sub}\left(\mathbf{P}_{2}\right)\right)$. Indeed, $\left\{a_{\omega}\right\} \leq$ $\left\{a_{k}\right\} \vee\left\{b_{\omega}\right\}$ for every $k \geq 1$, and these are all minimal nontrivial join covers with $\left\{a_{k}\right\} \notin \mathrm{D}_{k-1}^{*}\left(\operatorname{Sub}\left(\mathbf{P}_{2}\right)\right)$. Thus $\left\{a_{\omega}\right\} \notin \mathrm{D}^{*}\left(\operatorname{Sub}\left(\mathbf{P}_{2}\right)\right)$.

\section{Approximation by Finite lower bounded LatTices}

We say that a lattice $\mathbf{L}$ is approximated by lattices from a class $\mathcal{K}$ if $\mathbf{L}$ is embeddable into a direct product of lattices from $\mathcal{K}$.

In this section we consider those lattices that are subdirect products of finite lower bounded lattices, or equivalently, subdirect products of finitely generated lower bounded lattices. Example 3.3 shows that such a lattice need not be itself lower bounded, and Example 3.4 gives a lower bounded lattice which cannot be approximated by finite lower bounded lattices. However, as a consequence of Corollary 3.2 we have the following.

Lemma 5.1. A finitely generated lattice $\mathbf{L}$ is in $\operatorname{SP}\left(\mathcal{L B}_{\text {fin }}\right)$ if and only if $\mathbf{L} \in \mathcal{L} \mathcal{B}$.

Semenova [13] and Wehrung [14], independently, have found nice sufficient conditions for a lattice to be approximable by finite lower bounded lattices. 
It turns out that the class $\mathrm{SP}\left(\mathcal{L} \mathcal{B}_{\text {fin }}\right)$ admits a characterization very similar to that of $\mathcal{L} \mathcal{B}(k)$. For a lattice $\mathbf{L}$, let $\mathrm{D}_{0}^{+}(\mathbf{L})=\mathrm{D}_{0}(\mathbf{L})$, the set of (finitely) join prime elements of $\mathbf{L}$. Recursively, $x \in \mathrm{D}_{k+1}^{+}(\mathbf{L})$ if there exist finitely many finite subsets $R_{1}, \ldots, R_{m}$ of $\mathbf{L}$ such that $x \leq \bigvee R_{i}$ and $R_{i} \subseteq \mathrm{D}_{k}^{+}(\mathbf{L})$ for each $i$, and whenever $A$ is a finite subset of $\mathbf{L}$ with $x \leq \bigvee A$ nontrivially, then $R_{j} \ll A$ for some $j$.

For finitely generated lattices, $\mathrm{D}_{k}^{+}(\mathbf{L})=\mathrm{D}_{k}(\mathbf{L})$, while in general $\mathrm{D}_{k}^{+}(\mathbf{L}) \subseteq \mathrm{D}_{k}(\mathbf{L}) \subseteq$ $\mathrm{D}_{k}^{*}(\mathbf{L})$. To see that the containment may be proper, let $\mathbf{S}$ be the semilattice with infinitely many atoms $a_{i}(i \in \omega)$ and a zero element 0 . In the lattice $\mathbf{K}$ of all finite subsemilattices of $\mathbf{S}$, every one-element subsemilattice is in $D_{1}(\mathbf{K})$, but $\{0\}$ is not in $\mathrm{D}_{1}^{+}(\mathbf{K})$ because it has too many minimal nontrivial join covers. In fact, $\mathbf{S}$ is a subdirectly irreducible lattice in $\mathcal{L} \mathcal{B}(1)$.

As usual, we have $\left(\mathrm{D}_{k}^{+}(\mathbf{L})\right)^{\vee} \subseteq \mathrm{D}_{k+1}^{+}(\mathbf{L})$. Let $\mathrm{D}^{+}(\mathbf{L})=\bigcup_{k \geq 0} \mathrm{D}_{k}^{+}(\mathbf{L})$.

Let $\Delta_{k}^{+}(\mathbf{L})=\mathrm{D}_{k}^{+}(\mathcal{F}(\mathbf{L}))$ and $\Delta^{+}(\mathbf{L})=\mathrm{D}^{+}(\mathcal{F}(\mathbf{L}))$. Both of these sets have the WRP, so we can define homomorphisms $\delta_{k}^{+}: \mathbf{L} \rightarrow\left(\Delta_{k}^{+}(\mathbf{L})\right)^{\vee}$ and $\delta^{+}: \mathbf{L} \rightarrow\left(\Delta^{+}(\mathbf{L})\right)^{\vee}$ via

$$
\begin{aligned}
& \delta_{k}^{+}(x)=\bigcap\left\{F \in \Delta_{k}^{+}(\mathbf{L}): x \in F\right\} \\
& \delta^{+}(x)=\bigcap\left\{F \in \Delta^{+}(\mathbf{L}): x \in F\right\}
\end{aligned}
$$

with kernels denoted $\lambda_{k}^{+}$and $\lambda^{+}$, respectively. Clearly $\lambda^{+}=\bigcap_{k \geq 0} \lambda_{k}^{+}$.

Theorem 5.2. A lattice $\mathbf{L}$ is in $\operatorname{SP}\left(\mathcal{L B}_{\text {fin }}\right)$ if and only if $\lambda^{+}=0$ in $\operatorname{Con}(\mathbf{L})$.

The proof combines two lemmas.

Lemma 5.3. If $\mathbf{K}$ is a finite lattice, $h: \mathbf{L} \rightarrow \mathbf{K}$ is a homomorphism, and $a \in \mathrm{D}_{j}(\mathbf{K})$, then $h^{-1}(\uparrow a) \in \Delta_{j}^{+}(\mathbf{L})$.

Proof. For a finite lattice $\mathbf{K}$ and a lattice homomorphism $h: \mathbf{L} \rightarrow \mathbf{K}$, the map $\widehat{h}: \mathcal{F}(\mathbf{L}) \rightarrow \mathbf{K}$ defined by $\widehat{h}(F)=\bigwedge\{h(x): x \in F\}$ for all $F \in \mathcal{F}(\mathbf{L})$ is a complete lattice homomorphism. Note that for any $z \in \mathbf{K}$ we have $\widehat{h}(F) \geq z$ if and only if $F \geq h^{-1}(\uparrow z)$.

Now we proceed to prove the statement of the lemma by induction on $j$. If $a$ is join prime in $\mathbf{K}$ and $\bigvee_{i \in I} F_{i} \geq h^{-1}(\uparrow a)$, then $\widehat{h}\left(\bigvee_{i \in I} F_{i}\right)=\bigvee_{i \in I} \widehat{h}\left(F_{i}\right) \geq a$, whence $\widehat{h}\left(F_{s}\right) \geq a$ for some $s$, i.e., $F_{s} \geq h^{-1}(\uparrow a)$. Thus $h^{-1}(\uparrow a)$ is join prime in $\mathcal{F}(\mathbf{L})$, proving the case $j=0$.

So assume that the statement of the lemma holds for $j=k$, and let $a \in \mathrm{D}_{k+1}(\mathbf{K})$. There exist finitely many finite subsets $Q_{1}, \ldots, Q_{m}$ of $\mathbf{K}$ such that $a \leq \bigvee Q_{i}$ and $Q_{i} \subseteq \mathrm{D}_{k}(\mathbf{K})$ for each $i$, and whenever $A$ is a finite subset of $\mathbf{K}$ with $x \leq \bigvee A$ nontrivially, then $Q_{j} \ll A$ for some $j$. Let $R_{i}=\left\{h^{-1}(\uparrow x): x \in Q_{i}\right\}$. Now for each $i$ we have

$$
a \leq \bigvee Q_{i} \leq \bigvee_{G \in R_{i}} \widehat{h}(G) \leq \widehat{h}\left(\bigvee_{G \in R_{i}} G\right)
$$


whence $\bigvee R_{i} \geq h^{-1}(\uparrow a)$. Also, by induction $R_{i} \subseteq \Delta_{k}^{+}(\mathbf{L})$. Finally, assume that $h^{-1}(\uparrow a) \leq \bigvee B$ for some finite subset $B \subseteq \mathcal{F}(\mathbf{L})$. Then

$$
a \leq \widehat{h}\left(h^{-1}(\uparrow a)\right) \leq \widehat{h}(\bigvee B)=\bigvee_{F \in B} \widehat{h}(F)
$$

so that $Q_{i} \ll\{\widehat{h}(F): F \in B\}$. This implies $R_{i} \ll B$, as desired.

For an element $x \in \mathrm{D}^{+}(\mathbf{L})$, the join dependency closure of $x$ is the smallest subset $Y \subseteq \mathbf{L}$ such that $x \in Y$, and whenever $y \in Y$ and $Z$ is a minimal nontrivial join cover of $y$, then $Z \subseteq Y$.

Lemma 5.4. If $F \in \Delta_{j}^{+}(\mathbf{L})$, then the join dependency closure $\mathcal{U}_{F}$ of $F$ in $\mathcal{F}(\mathbf{L})$ is finite and has the WRP. Moreover, the lattice $\left(\mathcal{U}_{F}\right)^{\vee}$ is in $\mathcal{L} \mathcal{B}(j)$.

Proof. The finiteness of $\mathcal{U}_{F}$ is evident by induction, and the WRP follows from the definitions. One can prove by induction that if $F \in \Delta_{j}^{+}(\mathbf{L})$, then $F \in \mathrm{D}_{j}\left(\left(\mathcal{U}_{F}\right)^{\vee}\right)$. Therefore $\mathcal{U}_{F} \subseteq \mathrm{D}_{j}\left(\left(\mathcal{U}_{F}\right)^{\vee}\right)$, so that $\left(\mathcal{U}_{F}\right)^{\vee} \in \mathcal{L} \mathcal{B}(j)$.

Thus by Lemma 3.23, for each $F \in \Delta_{j}^{+}(\mathbf{L})$ there is the standard homomorphism $\varphi: \mathbf{L} \rightarrow\left(\mathcal{U}_{F}\right)^{\vee}$, which yields the following consequence.

Corollary 5.5. For any lattice $\mathbf{L}$ we have that $\mathbf{L} / \lambda^{+} \in \mathrm{SP}\left(\mathcal{L} \mathcal{B}_{\text {fin }}\right)$.

Acknowledgments. We want to thank Marina Semenova for her interest in this work and a fair amount of notes and suggestions sent to us.

\section{REFERENCES}

[1] K. Adaricheva, Semidistributive and co-algebraic lattices of subsemilattices, Algebra and Logic 27 (1988), 385-395; translated from Algebra i Logika 27, no. 6 (1988), 625-640.

[2] K. Adaricheva and V. Gorbunov, On lower bounded lattices, Algebra Universalis, 46 (2001), 203-213.

[3] K. Adaricheva and M. Semenova, Notes on "reasonable" lattices, manuscript, 2002.

[4] R. Freese, J. Ježek and J. B. Nation, Free Lattices, Mathematical Surveys and Monographs, 42, Amer. Math. Soc., Providence, 1995. viii+293 p.

[5] R. Freese and J. B. Nation, Covers in free lattices, Trans. Amer. Math. Soc. 288 (1985), 1-42.

[6] B. Jónsson and J. B. Nation, A report on sublattices of a free lattice, Contributions to Universal Algebra, North-Holland Pub. Co., Amsterdam, 1977, Coll. Math. Soc. János Bolyai, vol. 17, pp. 233-257.

[7] B. Jónsson and I. Rival, Lattice varieties covering the smallest nonmodular variety, Pac. J. Math. 82 (1979), 463-478.

[8] A. Kostinsky, Projective lattices and bounded homomorphisms, Pac. J. Math. 40 (1972), 111119.

[9] R. McKenzie, Equational bases and non-modular lattice varieties, Trans. Amer. Math. Soc. 174 (1972), 1-43.

[10] J. B. Nation, Some varieties of semidistributive lattices, Proceedings of the Charleston Conference on Lattice Theory and Universal Algebra (1985), Lecture Notes in Mathematics 1149, Springer-Verlag. 
[11] J. B. Nation, An approach to lattice varieties of finite height, Algebra Universalis 27 (1990), 521-543.

[12] M. V. Semenova, The lattices of suborders, Siberian Math. J. 40 (1999), 673-682.

[13] M. V. Semenova, On lattices embeddable into suborder lattices, to appear in Algebra and Logic.

[14] F. Wehrung, Sublattices of complete lattices with continuity conditions, to appear in Algebra Universalis.

Institute of Mathematics of SB RAS, Acad. Koptyug Prosp., 4, 630090, NovosiBIRSK, RUSSIA

Current address: Department of Mathematics, Harold Washington College, Chicago, IL 60601, USA

E-mail address: ki13ra@yahoo.com

Department of Mathematics, University of Hawail, Honolulu, Hi 96822, USA

E-mail address: jb@math.hawaii.edu 